\title{
A CFD study on the fluctuating flow field across a parallel triangular array with one tube oscillating transversely
}

Jorge Parrondo, Beatriz de Pedro*, Jesús Fernández-Oro, Eduardo Blanco-Marigorta

Energy Department, University of Oviedo, Spain

\begin{abstract}
This paper presents a novel CFD study on the fluctuations induced in water flow by a single vibrating tube in a parallel triangular array with pitch ratio of 1.57 . The simulations have been developed with a commercial code for the resolution of the 2D-URANS equations while allowing mesh updating at each time step, in order to incorporate forced oscillations in the transverse direction for a selected tube. After each simulation, the velocity, vorticity and pressure fields computed at successive time steps were FFT processed to obtain the corresponding distribution of fluctuations in amplitude and phase. This allowed for a convenient analysis of the disturbances inducted in the flow, which is considered key for the development of fluidelastic instability. According to these computations, during the oscillation cycle several vortices are formed around the vibrating tube, some of which are convected downstream at an approximately constant speed. That process determines the amplitude and phase of the velocity and pressure fluctuations throughout
\end{abstract}

\footnotetext{
*Corresponding author. E-mail address: pedrobeatriz@uniovi.es
} 
the domain, and, in particular, on the surface of the vibrating tube. Results are reported for a range of cross-flow velocities, from very low values up to a reduced gap velocity above 10 . The predictions obtained are compared to the results from some other studies in the literature, in particular to the experimental data on the propagation of velocity fluctuations reported by Khalifa et al. (2013a) and to the unsteady lift forces measured by Sawadogo and Mureithi (2014), in both cases for relatively similar configurations. Except for low flow velocity, the fluctuating lift forces result to be destabilizing due to the pressure pulsations on specific areas of the vibrating tube at the wake side, which behave as alternating stagnation regions for the periodic vortical flows induced by the oscillating tube.

Keywords:

Fluidelastic Instability, Fluid forces URANS model, Unsteady flow, Disturbance propagation, Tube arrays

\section{Introduction}

The development of self-excited vibrations in cylinder arrays subject to cross-flow such as in shell-and-tube heat exchangers, usually referred to as fluidelastic instability (FEI), can be triggered by either a fluid damping or a fluid stiffness controlled mechanism [Chen (1983), Paidoussis and Price (1988)]. The latter requires several degrees of freedom, i.e., it involves coupled oscillations of several tubes of the array, but the fluid-damping mechanism only requires motion of one single cylinder in one direction, with lower critical flow velocity for the oscillations in the transverse direction than streamwise. 
The phenomenon is attributed to the phase lag between the instantaneous fluid forces applied on the tubes and their position during each oscillation, though different origins have been proposed to explain that lag. For some authors, its origin lies on the adaptation process of the upstream flow to the tube motion, either due to fluid inertia effects in the main stream [Lever and Weaver (1982)] or because of fluid retardation in the vicinity of the stagnation points [Price and Paidoussis (1984)]. Instead, Paidoussis et al. (1984) associate that lag to the effect of viscous forces in the reorganization of the wake after the oscillating tube. More specifically, Granger and Paidoussis (1996) describe it as a memory effect due to the diffusion of thin vorticity layers from the oscillating tube surface into its boundary layer, to be finally convected downstream. Posteriorly, Meskell (2009) proposed a simple wake model to estimate Granger and Paidoussis's memory function, showing that that process of vorticity transport can produce the time delay of the fluidelastic force.

Lever and Weaver [Lever and Weaver (1982), Lever and Weaver (1986a), Lever and Weaver (1986b)] used the fluid inertia concept to build a quasianalytical model in which the main flow passes through the array along wavy channels or streamtubes. Given a transversely oscillating cylinder, the crossarea of the adjacent streamtubes is assumed to fluctuate too with a time lag that increases with distance. The application of simple 1D unsteady flow equations allows estimating the velocity and pressure fluctuations along the wavy channels, from which the fluid-dynamic forces on the tubes can be determined and used to establish a critical velocity for each configuration. As compared to other models, Lever and Weaver's is very attractive because 
it offers a simple way of applying the basic flow equations to explain the phenomenon while yielding predictions of critical velocity that are reasonably in-line with the trend of the experimental data for several situations. Posteriorly the model was extended to multiple flexible tubes by Yetisir and Weaver [Yetisir and Weaver (1993a), Yetisir and Weaver (1993b)] and more recently by Hassan and Weaver [Hassan and Weaver (2016a), Hassan and Weaver (2016b)] to account for both transverse and streamwise vibrations.

However, there have been few studies intended to verify how disturbances lag and decay while they are transmitted in the flow, despite its importance in the excitation mechanism for FEI as described in Lever and Weaver's model. The most remarkable experimental study corresponded to Khalifa et al. (2013a), who conducted detailed hot-wire measurements in the flow across a parallel triangular array with $\mathrm{P} / \mathrm{d}=1.54$ and one tube vibrating transversely due to damping-controlled FEI. This is very convenient because it allows isolating the source of the perturbations induced in the flow. Khalifa et al. (2013a) observed that velocity fluctuations originated near the flow separation region behind the oscillating tube. From that position the fluctuations propagated both up and downstream with a speed that was basically proportional to the gap velocity (lower speed upstream than downstream), though the fluctuation amplitude decayed quickly with increasing distance from the origin.

Other recent studies on this topic have been based on CFD simulations of the flow through arrays in which a single tube was forced to oscillate 
at a given amplitude and frequency. Hassan and El Bouzidi [Hassan and El Bouzidi (2012)], who considered a normal triangular array with $\mathrm{P} / \mathrm{d}=1.35$, obtained that the maximum velocity fluctuations were located close to both flow attachment and detachment regions on the vibrating tube, with a nearly constant velocity phase upstream. More recently, these authors [El Bouzidi and Hassan (2015)] focused on the fluctuations in the cross-area of the equivalent streamtubes through the same array. They concluded that the decay function used by Yetisir and Weaver (1993a) for the cross-area fluctuations is in general more realistic than the assumptions in the original Lever and Weaver's model, especially for low reduced velocities. Khalifa et al. (2013b) also performed CFD simulations intended to model the velocity fluctuations previously measured for a parallel triangular array [Khalifa et al. (2013a)]. They obtained good correlation between predictions and measurements, and besides they obtained improved estimations of critical velocity when using an empirical phase lag function in Lever and Weaver's model [Lever and Weaver (1986a), Lever and Weaver (1986b)]. Anderson et al. (2014) designed a numerical model inspired in Lever and Weaver's 1D theory to explore the development of the boundary layer alongside each cylinder in an in-line array with one oscillating tube. They obtained that the separation point oscillated with amplitude and phase (relative to tube motion) that depended non-linearly on the reduced flow velocity as well as on the excitation amplitude. They concluded that the time lag of the fluidelastic force has two components: one velocity-dependent component related to perturbations in the main stream and another amplitude-dependent component related to the boundary layer motion. 
This paper presents a novel CFD study on the fluctuations induced in the flow by a single vibrating tube in a parallel triangular array with $\mathrm{P} / \mathrm{d}=1.57$, not very different to the arrays tested Khalifa et al. (2013a) and Sawadogo and Mureithi (2014). The study is based on a 2D URANS model developed with the commercial code Fluent and complemented with an external software routine that imposed forced oscillations in the transverse direction for a selected tube. The methodology follows the previous work by de Pedro et al. (2016) for normal triangular arrays, who obtained remarkable agreement between predictions and experimental data on the time lag of the fluid-dynamic forces and, especially, on the critical velocity. After each simulation, the velocity, vorticity and pressure fields computed at successive time steps were FFT processed to obtain the corresponding distribution of fluctuations in amplitude and phase, thus allowing for a convenient analysis of the flow perturbations in the region of interest.

\section{CFD procedure}

A CFD methodology involving structure motion and dynamic re-meshing has been applied to simulate the unsteady flow through cylinder arrays with one single tube undergoing forced oscillations while subject to water crossflow. The procedure is based on 2D URANS computations with the Fluent 6.3 software. The choice of a 2D URANS formulation allows for a relatively low computational cost, but it entails a significant simplification with respect to a real 3D high turbulent flow as expected across packed cylinder arrays, 
because the effects of turbulence are condensed in averaged Reynolds stress terms. This means that only deterministic fluctuations can be computed, i.e. the coherence between the fluctuations predicted at any two positions will be unity regardless their distance, whereas in real flow it would decay with increasing separation. In consequence, the prediction with a URANS model of the fluid forces induced by a vibrating tube on the other cylinders in an array can be expected to lose reliability with increasing distance. Yet, a URANS model can be a convenient means to analyze the periodic flow perturbations induced around the oscillating tube and the subsequent fluiddynamic forces leading to damping-controlled FEI. This has already been shown in other numerical studies, for instance by de Pedro et al. (2016) for normal triangular arrays.

To incorporate the motion of the vibrating tube, the CFD model was complemented with a specially designed User Defined Function so that, at every time step, the position of the tube could be updated and the domain remeshed [de Pedro et al. (2016)]. For this purpose, a hexagonal region was defined surrounding the vibrating tube, in which triangular cells could either shrink or expand depending on the instantaneous tube position (Fig. 1-b). Around each tube, a special grid was defined composed by several layers of quadrilateral cells, the closer to the tube surface the smaller size. In the case of the vibrating tube, this ring of quadrilateral cells moved attached to the cylinder without undergoing deformation. For all the cases tested the $y^{+}$ parameter was equal or less than 1 . The array modeled corresponded to the parallel triangular geometry with $\mathrm{P} / \mathrm{d}=1.57$. This array had seven rows, with 
the vibrating tube located in the fourth row, and the calculation domain extended the equivalent to nine tube diameters both up and downstream (Fig. 1-a). The model contained about $4 \times 10^{5}$ cells.

Several preliminary series of computations were performed in order to select adequate calculation parameters including turbulence model (k- $\epsilon$ RNG), boundary conditions at the channel sides, mesh refinement and time step. Further details from these preliminary tests can be found in [de Pedro et al. (2016)]. In particular, full-slip guide plates were placed behind each tube of the last row, parallel to the main stream, in order to prevent the appearance of large-scale flow oscillations downstream. This was preferred to the option of truncating the domain at the centerline of the last cylinder row as used in other studies [Hassan et al. (2010)] in order to prevent affecting the disturbance propagation downstream by a constant pressure condition not too far from the vibrating cylinder. Besides, the time step for the computations now reported was set to 512 time steps per tube oscillation, which is about six times higher than the minimum value recommended by Hassan et al. [Hassan et al. (2010)] for equivalent simulations under forced vibration.

For each new case tested, dynamic simulations were left to progress over several oscillations until the successive maxima and minima of the lift coefficient on the vibrating tube and on the surrounding cylinders differed in less than $2 \%$ of the corresponding peak-to-peak amplitude. Then the instantaneous velocity and pressure fields at 64 instants covering one more tube oscillation were registered for posterior analysis. For this purpose, a new 
regular grid was defined for the region of interest, i.e. the region surrounding the vibrating tube including the wavy channels adjacent to it (see detail in Fig. 2). Typically, the nodes in this grid were separated $0.25 \mathrm{~mm}$ in both directions. In this regular grid, velocity and pressure time signals at each node were determined from adequate composition of the instantaneous flow fields previously computed with the CFD software. Besides, each instantaneous velocity field was processed to obtain the corresponding instantaneous vorticity distribution, so that vorticity time signals were also assigned to each node of the regular grid. Then FFT calculations were performed at each node to yield the amplitude and phase of velocity, vorticity and pressure at the vibration frequency and harmonics, as well as the average value. This procedure was applied systematically for different values of upstream velocity and vibration amplitude.

Figure 3 shows an example of the spectral distributions so obtained for the velocity and pressure amplitude at three reference locations close to the vibrating tube $\mathrm{TV}$, for a specific set of system parameters. Like in the case shown in Fig. 3, the amplitude of both velocity and pressure fluctuations for the second and higher order harmonics was always very small throughout the computational domain. Because of that, only predictions corresponding to the first harmonic (i.e., to the vibration frequency) are reported in this paper. 


\section{Pattern of the average flow}

Figure 4 shows the average flow field (streamlines and velocity and pressure distribution) obtained for a parallel triangular array with tube diameter $\mathrm{d}=12 \mathrm{~mm}$, pitch ratio $\mathrm{P} / \mathrm{d}=1.57$ and water cross-flow with upstream velocity $\mathrm{U}_{U}=0.21 \mathrm{~m} / \mathrm{s}$. The Reynolds number based on gap velocity was $\operatorname{Re}_{G}=6020$. Tube TV was oscillating transversely with amplitude $A=0.83 \%$ of tube diameter and frequency $\mathrm{f}=7.81 \mathrm{~Hz}$. The resulting reduced gap velocity was $\mathrm{U}_{R G}=5.33$. This was considered as the reference case.

As expected, the flow pattern is characterized by wavy stream channels between tube lines, with low velocity wake regions between tubes of the same line. The pattern is notoriously symmetric with respect to the horizontal line through the centers of tubes T1, TV and T4, though the domain (Fig. 1-a) is not strictly symmetric. After the second row, the stream channels are seen to attach to each tube surface at about $\beta_{1} \sim 35^{\circ}$ ahead of the tube top or bottom, whereas detachment starts just at $\beta_{2} \sim 5^{\circ}$ after of those positions. No significant variations were found in this velocity pattern when modifying the flow velocity or the vibration amplitude.

These wavy lanes resemble the streamtubes considered in Lever and Weaver's theory. Though they assumed symmetrical locations for the flow attachment and detachment from each cylinder (i.e., $\beta_{1} \sim \beta_{2}$ ) [Lever and Weaver (1986a)], the input data they proposed for parallel triangular arrays with $\mathrm{P} / \mathrm{d}=1.6$ [Lever and Weaver (1986b)] corresponds to $\beta_{1} \sim \beta_{2}=18.5^{\circ}$; this 
gives an angular extension for the region of contact between cylinders and stream tubes that is close to the current prediction of $\beta_{1}+\beta_{2} \sim 40^{\circ}$ (Fig. 3). Nonetheless, the current predictions are in line with the values $\beta_{1}=40^{\circ}$ and $\beta_{2}=10^{\circ}$ suggested by Yetisir and Weaver [Yetisir and Weaver (1993b)] for parallel triangular arrays, based on a previous flow visualization study by Scott (1987).

The pressure pattern of Fig. 4-c shows the progressive decay of pressure through the array, from highest values at the stagnation point on the tubes of the first and second rows. Along the stream channels, pressure is seen to decrease locally round the top and bottom portions of each cylinder in the array, approximately at the detachment regions, whereas the kinetic energy rises up in the mid-stream. In the wake regions, pressure is mostly determined by the pressure in the adjacent stream tubes and so it is rather uniform until the stagnation points on the next tube downstream.

\section{Velocity fluctuation field}

Figure 5 presents the instantaneous fluctuation of the velocity vectors when the oscillating tube TV is located at four different positions during one cycle. They were obtained for the same conditions of the reference case by subtracting the average velocity vectors (modules as in Fig. 3) from the instantaneous values. Only one out of 64 vectors is shown in Fig. 5.

It is seen that the motion of tube TV induces vortexes in the wake regions 
T1-TV and TV-T4 with alternating sense of rotation and highest intensity approximately when TV is at maximum speed, i.e., when it passes through the neutral position either upwards $\left(0^{\circ}\right)$ or downwards $\left(180^{\circ}\right)$. These vortices are mostly related to the fluid areas alternatively displaced in and out by tube TV as it moves up and down during the oscillation cycle. For example, when $\mathrm{TV}$ is at $0^{\circ}$ going upwards (first graph of Fig. 5), the fluid displaced from the top of TV plus the fluid suctioned from its bottom side and the fluid layers dragged upwards form a counter-clockwise vortex between T1 and TV, as well as a clockwise vortex between TV and T4. These two vortices are not wholly symmetric because the main stream imposes different flow conditions up and downstream from TV.

Besides, alternating vortices are also induced in the region of the wavy channels adjacent to tube TV, with highest intensity approximately when TV is at maximum displacement $\left(90^{\circ}\right.$ and $\left.270^{\circ}\right)$. These vortices are related to the periodic changes of cross-section in the wavy channels and the corresponding changes in hydraulic resistance. When, for instance, TV is at bottom position $\left(270^{\circ}\right.$, fourth map in Fig. 5), the channel cross-section below TV is lowest, part of the incoming stream between $\mathrm{T} 1$ and $\mathrm{T} 2$ is diverted upwards through the T1-TV passage and part of the stream above TV is diverted downwards through the TV-T4 passage. As a result, at that instant the velocity fluctuation alongside the bottom of $\mathrm{TV}$ is negative (it goes upstream) and a counter-clockwise vortex is located below TV. Unlike the former vortices in wake regions, these new vortices do not remain fixed but are progressively convected downstream while TV oscillates. Consider the clockwise vortex 
induced below TV when it is at top position $\left(90^{\circ}\right)$. When TV comes back through neutral position $\left(180^{\circ}\right)$ that vortex has already overpassed the top of tube T3, then it is right below T4 when TV reaches bottom position $\left(270^{\circ}\right)$, and finally, it is seen to have overpassed T4 when TV goes again through neutral position $\left(0^{\circ}\right)$.

The vectors with highest velocity are seen to be placed alongside the top and bottom surfaces of tube TV, but this is more readily appreciated in Fig. 6 , which shows the distribution of the velocity fluctuations for the first harmonic, i.e., at the vibration frequency, for the configuration of the reference case (the same as in Figs. 3 to 5). Figure 6-a shows the fluctuation amplitude and, like Fig. 4, it is mostly symmetric with respect to the horizontal line through the center of TV. Analogously, Fig. 6-b presents the phase of the velocity fluctuation with respect to the displacement of TV. In this case the phase distribution of Fig. 6-b is mostly anti-symmetric. The sign convention is that a positive phase expresses delay with respect to TV position, and the latter increases upwards (i.e. towards tubes T5 and T6). Therefore, a phase of, say, $10^{\circ}$, would mean that the highest value is achieved slightly after TV has passed through top position (i.e. $90^{\circ}$ in Fig. 5).

Figure 6-a confirms that the maximum fluctuation values correspond to the region of the main stream adjacent to the boundary layer of the vibrating tube. Highest values are of the order of $15 \%$ of the gap velocity, which is about twice the tube velocity amplitude for this particular case. On the left side of TV, several zones appear with some appreciable velocity fluc- 
tuation that are related to the alternating secondary flows in the T1-TV passage. They include zones surrounding the attachment of the main stream on TV. But indeed the right side of TV is more relevant: once the flow separates, high amplitude values still remain along the boundary region between main stream and cylinder wakes. This behavior is in line with Granger and Paidoussis' explanation for what they call flow memory effect [Granger and Paidoussis (1996)], due to the diffusion and subsequent convection of thin vorticity layers from the oscillating tube surface. However, it can also be attributed to the shift of the separation points while TV oscillates (Anderson et al. (2014)). Actually both attachment and separation points undergo some small shift in the present case (about $0.7^{\circ}$ and $1.4^{\circ}$ respectively between top and bottom position), but across the detached shear layer the gradient of average velocity is higher and so the velocity fluctuations are more intense downstream from flow separation.

Consider the case of experimental measurements with hot wire, like the tests performed by Khalifa et al. for a similar configuration [Khalifa et al. (2013a)]. Certainly, it would be difficult to get close enough to a tube surface that is vibrating as to measure the highest values suggested in Fig. 6-a. If measurements were to be taken starting at some safety distance from the vibrating tube, the highest values would correspond to the region of separated flow after the cylinder, i.e., slightly downstream from the detachment point. This was in fact the result obtained by Khalifa et al. (2013a) from their tests and it is consistent with the present computations. 
On the other hand, the map of Figure 6-b shows that the velocity phase is about $0^{\circ}$ at the bottom of $\mathrm{TV}$ and $180^{\circ}$ on top, but across the respective main stream channels the phase changes $180^{\circ}$. This is due to the vortices formed in the main channels. Consider the stream channel below TV when it is at top position, as seen in the second graph of Fig. 5. The velocity fluctuation alongside TV is highest and its direction is downstream, so that the modulus of the velocity vector is highest too and the phase delay with respect to the position of $\mathrm{TV}$ is nearly zero. At the opposite side of the stream channel (from T2 to T3) the velocity fluctuation is also highest but its direction is upstream, and so at that instant the modulus of the velocity vector is lowest and the phase delay is close to $180^{\circ}$. In contrast, in the middle of the channel, which corresponds to the core region of the vortices induced in the main stream, Fig. 6-b shows that the phase is not uniform but increases rapidly upstream, until the passage between T2 and TV. Further upstream the phase reduces somewhat and becomes uniform. Finally, downstream from TV, the phase is seen to increase progressively at both sides of the stream channel, though there is an abrupt change of phase across the central line. This is indicative of the vortex convection process shown in Fig. 5. In any case, those phase distributions mean that velocity perturbations are perceived after a delay that increases with distance from TV. This is in agreement with the results obtained by Khalifa et al. (2013a).

Finally, the fluctuations predicted at any position in the domain are totally correlated with the tube motion despite the separation distance. This unrealistic behavior is a consequence of the URANS formulation, as men- 
tioned in Section 2. In real turbulent conditions the vortical structures would progressively transform into smaller eddies, thus weakening the vortex convection downstream. However, around TV these vortices are continuously formed and sustained by the oscillations of the cylinder TV itself, despite the turbulence intensity in the main stream. Hence, in the neighborhood of $\mathrm{TV}$, it can be expected that a more realistic turbulent environment would only produce secondary effects on the unsteady flow.

\section{Velocity fluctuations along fluid lines}

For a better analysis of the predictions obtained, data is now presented for specific wavy fluid lines along the stream channels. These fluid lines have been generated automatically as a function of a special parameter $\mathrm{k}_{R}=[0 \ldots 1]$ that determines the distance from the vibrating tube: the line with $\mathrm{k}_{R}=0$ is adjacent to T1-TV-T4, whereas the line with $\mathrm{k}_{R}=1$ is adjacent to T2-T3. Data is presented as a function of a curvilinear coordinate $\mathrm{s}^{*}$, which has been normalized so that it takes values of $-2,-1,0,1$ and 2 respectively for the locations in front of the center of tubes T1, T2, TV, T3 and T4. Figure 7 presents the amplitude and phase of velocity fluctuations for three fluid lines close to tube TV, especially the first one (red dots, with $\mathrm{k}_{R}=0.05$ ). The system parameters are those of the reference case (Section 3). The phase has been expressed in terms of time delay normalized by the oscillation period $\left(t^{*}\right)$.

For comparison purposes Fig. 7 also shows the experimental data ob- 
tained by Khalifa et al. [6] by traversing a hot-wire probe along the middle of a wavy channel in an array with a relatively similar configuration. In particular, those measurements correspond to $\mathrm{P} / \mathrm{d}=1.54$ (thus gap is $\sim 5 \%$ lower than in the current model), air as flowing fluid and a reduced gap velocity in the range $\mathrm{U}_{R G}=[6-8]$. The Reynolds number based on gap velocity was in the order of $3 \times 10^{4}$, which is about five times higher than in the current simulations. Besides, Khalifa et al. did not impose a forced transversal vibration on a tube of the fourth row, but let a single tube (2 DOF) of the third row develop fluidelastic instability. Despite these differences, Khalifa et al's data can still be considered a reference for comparison with the current predictions.

Figure 7-b shows that, as discussed in the previous section, the highest velocity fluctuations take place alongside the tube surface, i.e., for the line $\mathrm{k}_{R}=0.05$. For increasing radial distance from TV, those high fluctuations become lower and the maximum values take place progressively further downstream. The experimental data [Khalifa et al. (2013a)] present maximum values of the order of those for the line $\mathrm{k}_{R}=0.1$, though the location $\mathrm{s}^{*}$ is slightly downstream. As discussed in the previous section, these experimental data can be considered consistent with the current predictions. This is also supported by the local increment in velocity fluctuation registered by both experimental data and predictions at about $\mathrm{s}^{*}=-0.6$, where flow is nearing the attachment region on TV.

Regarding the time delay of the velocity fluctuations (Fig. 7-c), the pre- 
dictions for the line $\mathrm{k}_{R}=0.05$ show that $\mathrm{t}^{*}$ is about zero along the surface of tube TV (around $\mathrm{s}^{*}=0$ ), and that it increases quite progressively downstream until $\mathrm{s}^{*}>2$. Changing to lines $\mathrm{k}_{R}=0.1$ and 0.25 makes the position for minimum delay shift progressively downstream, but for $\mathrm{s}^{*}>0.5$ the delay values are very similar to those of line $\mathrm{k}_{R}=0.05$. As already mentioned, this reflects disturbance propagation downstream and is in agreement with Khalifa et al.'s experimental data.

Khalifa et al.'s data also reveal clear disturbance propagation upstream, with a quite continuous increment in phase until $\mathrm{s}^{*}=-2$, despite the fluctuation amplitude is small. In comparison, the current computations reflect a more complex pattern upstream. For the line $\mathrm{k}_{R}=0.25$, which can be considered representative of the central part of the wavy channel, the time delay increases rapidly upstream until a value for $\mathrm{t}^{*}$ above 0.9 at about $\mathrm{s}^{*}=-0.7$, but afterwards it reduces to $\mathrm{t}^{*}=0.65$ with a relative minimum at about $s *=-$ 1.2. In contrast, the line $\mathrm{k}_{R}=0.05$, which passes very close to tube $\mathrm{TV}$, exhibits a continuous reduction of $t^{*}$ upstream until that minimum, though a sharp jump, equivalent to one oscillation period, has been artificially introduced before for ease of comparison with the other curves. The line $\mathrm{k}_{R}=0.1$ presents mixed features. This behavior is to be associated to the periodic secondary flow induced in the wake region between T1 and TV, since, according to the velocity maps of Fig. 5, it is significantly protruding into the main wavy channels. Presumably, had the effects of that secondary flow been less prominent, the delay of the velocity fluctuations along the central part of the wavy channel might have increased upstream with a less steep slope, 
more alike to Kahlifa et al.'s measurements. However it is uncertain to what extent that discrepancy can be attributed to the different conditions between experiments and simulations, or to the approximations of the computational model, including the assumptions implicit in a 2D URANS formulation.

A series of simulations was conducted varying the vibration amplitude from $0 \%$ to $8 \%$ of tube diameter while keeping the remaining parameters constant. No significant flow fluctuation could be detected if the vibration amplitude was zero (non-oscillating tube). Even for the highest vibration amplitude the tube velocity was an order of magnitude less than the gap velocity. The predictions obtained, which are presented in Fig. 8 for the fluid line with $\mathrm{k}_{R}=0.2$, show that the curves of velocity amplitude virtually collapse if they are normalized by the tube velocity. Besides, the time delay curves show no significant effect either. This proportionality between flow fluctuations and vibration amplitude for not very large oscillations is in fact a basic assumption in the models used to predict fluidelastic instability inception, and it is also well supported by experimental measurements (for instance Khalifa et al. (2013a)). In any case, this result confirms that the CFD predictions were not affected by excessive cell distortion during the process of mesh stretching and compression.

\section{Variation of flow fluctuations with flow velocity}

In the maps of Figs. 5 and 6 , the velocity fluctuations up and downstream from TV are very different, due to the relative high momentum in the wavy 
channels. Reducing the flow velocity without modifying the tube vibration weakens the influence of the main stream, and a more symmetrical pattern is to be expected between up and downstream. This is well characterized by the amplitude and phase maps presented in Fig. 9 for an upstream velocity $\mathrm{U}_{U}=0.03 \mathrm{~m} / \mathrm{s}\left(\mathrm{U}_{R G}=0.76, \mathrm{Re}_{G}=860\right.$, other parameters as in Fig. 3).

The unsteady flow is now dominated by the fluid directly pushed in and out by TV and there is very little difference in amplitude distribution between up and downstream. As in Fig. 6, the highest fluctuations in Fig. 9 take place along the top and bottom of $\mathrm{TV}$, with phases equal to $180^{\circ}$ and $0^{\circ}$ respectively (similar to Fig. 6). However, around TV there are other eight zones of relatively high amplitude and phases switching between $-90^{\circ}$ and $90^{\circ}$, i.e., their highest values are achieved when TV is passing through the neutral position at maximum speed either upwards or downwards. These regions, which can also be traced in the maps of Fig. 6, just reflect the alternating flows induced in the zones T1-TV and TV-T4. Consider the instant when TV is at neutral position moving upwards. Apart from some layers of fluid dragged upwards, the relevant flows are formed downwards at both sides of TV to transport fluid being removed from the top of TV to the space being liberated at the bottom. At the left-bottom region from TV, for instance, the combination of that flow with the continuous stream coming from the left side makes that the velocity modulus is maximum at that instant, i.e. it is $90^{\circ}$ ahead with respect to the displacement of $\mathrm{TV}$, and so the phase delay is $-90^{\circ}$ according to the adopted sign convention. 
In particular, those alternating downward and upward flows happen to produce velocity fluctuations with similar high amplitude at the regions close to both flow attachment $\left(\mathrm{s}^{*} \sim-0.65\right)$ and detachment $\left(\mathrm{s}^{*} \sim 0.65\right)$. This is so because, contrary to the case of Fig. 6, now the gradient of average velocity across the detached shear layer is low. The other zones with high amplitude, which are next to TV at the left and right sides, are related to the secondary flows induced in the inter-cylinder passages by the layers of fluid dragged by TV.

Finally, no vortex convection along the wavy channels is identified in Fig. 9 for such a low flow velocity. In fact, the phase distribution downstream from TV is rather uniform, as well as upstream, with about a $180^{\circ}$ shift between both sides. Khalifa et al. (2013b), who obtained similar results from their computations, suggested that disturbance propagation for low reduced velocity has an acoustic nature as opposed to the case of higher reduced velocity, for which propagation is determined by vorticity convection. They concluded that the acoustic mechanism would be dominant for $\mathrm{U}_{R G} \leq 2$, whereas in the present case vortex convection downstream could be detected for $\mathrm{U}_{R G} \geq 1.53$.

Figure 10 shows the velocity fluctuations along a fluid line close to tube $\mathrm{TV}\left(\mathrm{k}_{R}=0.2\right)$ for five flow velocities ranging from $\mathrm{U}_{U}=0.03 \mathrm{~m} / \mathrm{s}$ to $0.42 \mathrm{~m} / \mathrm{s}$ $\left(\mathrm{U}_{R G}=[0.76 \ldots 10.7], \mathrm{Re}_{G}=[860 \ldots 12000]\right)$. The velocity amplitude, which has been normalized by the gap velocity $\mathrm{U}_{G}$, shows a very similar pattern. Only for the low velocity range, further velocity reductions result in higher fluctu- 
ations at about $\mathrm{s}^{*}=-0.65$, so that for $\mathrm{U}_{R G}=0.76$ there is an almost symmetric pattern upstream and downstream as already reflected in Fig. 9. This is in line with the results obtained from the numerical studies by Hassan and El Bouzidi (2012) for a normal triangular array and by Khalifa et al. (2013b) for a parallel triangular array.

Figure 10 also shows the phase of the velocity fluctuations at each location in terms of the normalized time delay relative to the displacement of tube TV. Again, the curves for flow velocities with $\mathrm{U}_{R G}>0.76$ exhibit a similar behavior, though increasing the flow velocity brings about a less steep slope for the time delay evolution downstream from $\mathrm{s}^{*}=0$. A slope with lower steepness represents a higher propagation speed, which is consistent with the fluctuations being convected downstream from tube TV. For $\mathrm{U}_{R G}=0.76$, which is the same case of the maps of Fig. 9, there is no vortex convection: fluctuations far from TV are mostly due to fluid stirring around the fixed tubes such as T4, and so the phase does not increase progressively downstream.

\section{Speed of disturbance propagation}

As already described in Section 4, the disturbances along the stream channels are associated to the vortices formed in the main stream while tube TV vibrates and their subsequent convection downstream. The superposition of the vortices with the main flow makes that, across the stream channels, at one half the instantaneous velocity increases while it decreases at the other 
half. Because of this, the phase of the velocity fluctuations shown in Fig. 6-b exhibits a sharp change in value across the stream channels downstream from TV. In consequence, analyzing the propagation of flow disturbances from velocity fluctuations along a fluid line may be dependent on the specific fluid line selected. To reduce that fluid line dependence, vorticity fluctuations can be considered more convenient to estimate the speed of disturbance propagation.

Figure 11 presents the distribution of the phase of the vorticity fluctuations for the same configuration considered in the preceding sections 3-5. The sign convention is that vorticity is positive for counter-clockwise rotation and the phase represents delay relative to the position of tube TV, which is positive upwards. This produces a map that is virtually symmetric with respect to the T1-TV-T4 axis. The regions upstream, where the phase shows a granular appearance, possess very low vorticity amplitude. Downstream from TV, however, where the vorticity amplitude is larger, the phase shows a well-established increasing trend. As expected, the vorticity phase does not show the abrupt change of the velocity fluctuations of Fig. 6-b across the stream channels. Still, the boundaries imposed by tubes T3 and T4 (or T5 and T4) are seen to produce a higher growth rate close to their surfaces. This is because they contribute to slow down the vortex motion, so that the propagation speed of disturbances is highest along the middle of the stream channels.

Figure 12 shows the phase delay of the vorticity fluctuations downstream 
along the central fluid line $\left(\mathrm{k}_{R}=0.5\right)$ for several flow velocities. In all cases the phase is about $180^{\circ}$ around $\mathrm{s}^{*}=0$, which means that when TV is at top position (equivalent to $90^{\circ}$ during the oscillation cycle of Fig. 5) the vortex formed below TV is rotating clockwise with highest intensity (as shown in the second graph of Fig. 5). Downstream from $\mathrm{s}^{*}=0.5$, i.e., beyond the region where the vortices are formed, the delay is seen to increase quite progressively, in line with the previous results. As in Fig. 10-b, the higher the flow velocity the less steep the slope of the vorticity delay, i.e., the higher the speed of disturbance propagation downstream.

For each flow velocity, the speed of disturbance propagation was estimated by performing a linear fit of the vorticity time delay along that central fluid line $\left(\mathrm{k}_{R}=0.5\right)$ between $\mathrm{s}^{*}=0.6$ and $\mathrm{s}^{*}=2.4$, i.e., covering nearly two rows downstream from TV. This always gave at least 20 nodes for the regression analysis. The resulting determination coefficient $R^{2}$ was always above 0.97 , including a flow velocity as low as $\mathrm{U}_{R G}=1.53$ (no vortex convection could be traced for still lower $\mathrm{U}_{R G}$ ). Figure 13 presents the results obtained in terms of the ratio between the speed of disturbance propagation, $\mathrm{u}_{D}$, and the gap velocity, $\mathrm{U}_{G}$. It is seen that increasing the flow velocity until $\mathrm{U}_{R G} \sim 5$ makes the $\mathrm{u}_{D} / \mathrm{U}_{G}$ ratio reduce from nearly 1 to about 0.4 , and it remains around that value for higher flow velocities. This behavior of the $\mathrm{u}_{D} / \mathrm{U}_{G}$ ratio can be attributed to the pushing effect of the oscillating flow on the moving vortexes, which is only significant for low cross-flow velocities, and to the partial blockage effect of T3, T4 and following cylinders on the convected vortices, which restrain their motion to a speed well below the gap velocity. 
These results can be compared to the data reported by Khalifa et al. (2013a), based on hot wire measurements in wind tunnel for an array similar to that of the present CFD study (see Section 5) operating in the range of $\mathrm{U}_{R G}$ between 6 and 8 . They obtained that the $\mathrm{u}_{D} / \mathrm{U}_{G}$ ratio for disturbance propagation upstream and downstream from a vibrating tube was about 0.42 and 0.52 respectively. In the current study no constant speed propagation could be clearly determined upstream, but the results obtained for downstream propagation in the same range of $\mathrm{U}_{R G}$ give a value of $\mathrm{u}_{D} / \mathrm{U}_{G} \sim 0.4$, which is not far from Khalifa et al.'s data.

\section{Pressure fluctuations on the vibrating tube}

Figure 14 presents the fluctuating pressure field, in amplitude and phase, corresponding to the reference case of Figs. $4-6\left(\mathrm{U}_{R G}=5.34, \mathrm{~A} / \mathrm{d}=0.83 \%\right.$, $\mathrm{f}=7.81 \mathrm{~Hz}$ ). As expected, the pressure phase exhibits obvious anti-symmetry with respect to the line through the centers of T1, TV and T4. Maximum amplitude values correspond to the regions of flow most directly displaced by the tube motion, i.e. the regions labeled $\mathrm{A}$ and $\mathrm{A}^{\prime}$ at bottom and top of TV respectively (Fig. 14-a), despite the velocity fluctuations there are high only at the tube surface itself (Fig. 6-a). The phase with respect to tube position is about $0^{\circ}$ at the bottom $(\mathrm{A})$ and $180^{\circ}$ on top $\left(\mathrm{A}^{\prime}\right)$. This means that when tube $\mathrm{TV}$ is at maximum displacement upwards (equivalent to $90^{\circ}$

during the oscillation cycle of Fig. 5) pressure is highest at the region A and lowest at A'. That position of TV corresponds to the second map of velocity 
vectors of Fig. 5, which shows some stagnation being induced below TV by the clockwise vortex. Thus the opposite situation of lowest pressure below TV (region A) takes place when tube TV is at maximum displacement downwards (equivalent to $270^{\circ}$ during the oscillation cycle of Fig. 5), where the vortex below TV (which is now anti-clockwise) induces high velocity values in the negative direction as seen in the fourth map of Fig. 5.

Besides, Fig. 14-a also shows high pressure fluctuation values at the regions denoted as B and B', which are about $45^{\circ}$ upstream from A and A' respectively. Approximately, they correspond to the stagnation points of the average flow on TV (Fig. 3), where the average pressure reaches a relative maximum too (Fig. 4). At the region B, the phase of the pressure fluctuation is slightly less than $180^{\circ}$. Therefore, pressure there reaches the highest value when TV is close to maximum displacement downwards (close to $270^{\circ}$ during the oscillation cycle of Fig. 5), i.e. when the width of the T2-TV passage is lowest. The fourth vector map of Fig. 5 shows that the resulting partial blockage on the in-coming flow produces significant flow deviation upwards through the gap between T1 and TV. Similar observations can be made for the region B', where the pressure phase is slightly less than $0^{\circ}$ and thus maximum values are achieved when $\mathrm{TV}$ is close to maximum displacement upwards.

Finally, significant pressure fluctuations on tube TV can be observed too at the locations C-C', which are close to the flow detachment positions from TV (Fig. 14). In fact, those two regions can be seen to extend across the 
main streams towards tubes T3 and T5 respectively, with little change in amplitude or phase. The pressure phases are about $-90^{\circ}$ at $\mathrm{C}$ and $90^{\circ}$ at C', which means that, when the tube velocity is highest upwards (i.e., when tube TV is at $0^{\circ}$ during the oscillation cycle of Fig. 5), the instantaneous pressure is highest at $\mathrm{C}$ and lowest at C'. At that instant, the first graph of Fig. 5 shows a large clockwise vortex formed between TV and T4 as well as an anti-clockwise vortex above T3. Both vortices induce a high backwards relative velocity in the main stream towards the separation region from $\mathrm{TV}$, so that the total downstream velocity reduces and the instantaneous pressure increases. In particular, when tube $\mathrm{TV}$ is at $0^{\circ}$ during the oscillation cycle, the area $\mathrm{C}$ behaves as a stagnation region for that secondary flow and so pressure at $\mathrm{C}$ is highest. The opposite behavior takes place when $\mathrm{TV}$ is at maximum velocity downwards, i.e., at $180^{\circ}$ during the oscillation cycle of Fig. 5.

These results can be compared with the instantaneous pressure maps reported by Khalifa et al. (2013b), obtained from CFD simulations for a similar array with one oscillating tube (configuration geometry as in the experimental tests by Khalifa et al. (2013a)). They imposed a reduced gap velocity $\mathrm{U}_{R G}=4$, which is $25 \%$ lower than the value $\mathrm{U}_{R G}=5.34$ used for Fig. 14, but despite that the agreement with the current predictions of pressure fluctuation is remarkable.

To appreciate how those pressure fluctuations depend on the cross-flow velocity, Fig. 15 shows the pressure amplitude and phase along the fluid 
line with $\mathrm{k}_{R}=0.05$, which is nearly attached to tube $\mathrm{TV}$, for several values of the reduced gap velocity from $\mathrm{U}_{R G}=2.93$ to 8.01. In all cases the vibration amplitude of tube $\mathrm{TV}$ was $\mathrm{A} / \mathrm{d}=0.83 \%$ and the frequency was $\mathrm{f}=7.81$ Hz. The pressure amplitude, which has been normalized by the dynamic pressure associated to the gap velocity $\mathrm{U}_{G}$, always exhibits a clear maximum very close to $\mathrm{s}^{*}=0$, which corresponds to the zone below A in Fig. 14. The magnitude of the normalized amplitude at that position is seen to decrease for increasing values of $\mathrm{U}_{R G}$, whereas its phase (relative to tube TV position) is always very close to zero (as seen in Fig. 14-b).

Except for the lowest cross-flow velocity, another relative maximum appears at about $\mathrm{s}^{*}=-0.6$, i.e., at the region $\mathrm{B}$ in Fig. 14. Increasing $\mathrm{U}_{R G}$ makes that the relative amplitude in that position approaches the value at $\mathrm{s}^{*}=0$, while the phase evolves from above $90^{\circ}$ to almost $180^{\circ}$ for $\mathrm{U}_{R G}=8.0$. At the other side, at $\mathrm{s}^{*} \approx+0.6$ (i.e., the zone close to $\mathrm{C}$ in Fig. 14) the amplitude is always low since it is reducing towards a minimum beyond $s^{*}=1$, while the phase varies from about $-60^{\circ}$ to $-120^{\circ}$ when increasing $\mathrm{U}_{R G}$ from 2.93 to 8.01. Finally, approximately between $\mathrm{s}^{*}=0.8$ and $\mathrm{s}^{*}=1.8$, all the phases augment in about a full $360^{\circ}$ period, i.e., all the curves finish in phase values similar to those at $\mathrm{s}^{*} \approx+0.6$ as shown explicitly for $\mathrm{U}_{R G}=2.93$. In conclusion, the pressure fluctuations on tube TV exposed in the maps of Fig. 14 can be considered sufficiently representative for a wide range of cross-flow velocities, with the exception of the region close to $\mathrm{B}$ for low values of $\mathrm{U}_{R G}$. 


\section{Fluctuating lift force and discussion}

Since the pressure fluctuations on TV at the zones A and A' (Fig. 14) are respectively in phase and $180^{\circ}$ out of phase with the tube position, the associated dynamic force on TV does not have a component in phase with the tube velocity. This also stands for the pressure fluctuations at the zones $\mathrm{B}$ and $\mathrm{B}$ ' for high values of $\mathrm{U}_{R G}$. Therefore, they cannot really explain the self-excitation of vibrations due to damping-controlled fluidelastic instability. In comparison, the pressure fluctuations on the $\mathrm{C}$ and $\mathrm{C}$ ' areas of tube TV clearly contribute to create a force component that is in phase with tube velocity, as shown below.

Figure 16 presents the amplitude $\mathrm{C}_{L, A}$ and phase $\mathrm{C}_{L, P h}$ of the fluctuating lift coefficient on tube $\mathrm{TV}$ as a function of $\mathrm{U}_{R G}$ (again the vibration amplitude and frequency remained constant). This lift coefficient represents the dynamic lift force due to both pressure and viscous stress distributions around TV, divided by the tube vibration amplitude and by the dynamic pressure associated to the gap velocity. Together with the values obtained from the current CFD study, Fig. 16 also shows a continuous line that represents the data measured by Sawadogo and Mureithi (2014) for the case of a parallel triangular array with $\mathrm{P} / \mathrm{d}=1.5$ (gap is $12 \%$ lower than with the present $\mathrm{P} / \mathrm{d}=1.57$ ), single phase flow and one single tube undergoing forced vibrations transversely, like in the present case. Their highest Reynolds number was about six times larger than in the current CFD simulations, and the oscillating tube was located in the seventh row. Posteriorly Li and Mureithi (2017) obtained similar results for another array with the same pitch and the 
vibrating tube located in the eleventh row. For Fig. 16, the original data of lift coefficient and reduced velocity reported by Sawadogo and Mureithi (2014) have been expressed relative to the gap velocity instead of the pitch velocity.

Figure 16-a shows that increasing $\mathrm{U}_{R G}$ brings about a quick reduction in amplitude until values of the order of 0.01 for $\mathrm{U}_{R G}>5$. Despite the difference in pitch and Reynolds range, the quantitative agreement of the predictions with the experimental data is noteworthy, with the computed values being about 10\% higher than measurements. On the other hand both measured and computed phases (expressing delay relative to tube TV position) are seen in Fig. 16-b to start with nearly zero values for very low $\mathrm{U}_{R G}$, then increase until a maximum at about $\mathrm{U}_{R G}=1.5$ for the predictions and $\mathrm{U}_{R G}=4.5$ for the measurements, and finally decay respectively towards $-135^{\circ}$ and $-90^{\circ}$ at $\mathrm{U}_{R G} \approx 10$. Predictions and measurements of the lift phase are not so close to each other as the lift amplitude, but the qualitative agreement is still good. Since the tube velocity is $90^{\circ}$ ahead of tube position, the values of phase delay relative to tube velocity can be obtained by adding $90^{\circ}$ to the values of Fig. 16b. This means that, in order to obtain a positive lift component in phase with tube velocity, the phase delay relative to tube position must lie between $-180^{\circ}$ and $0^{\circ}$. In the present case this occurs for values of $\mathrm{U}_{R G}>2.3$ (predictions) or $\mathrm{U}_{R G}>5$ (measurements). Above these limits, in consequence, damping controlled fluidelastic instability in the transverse direction can be expected for the corresponding configurations. This is consistent with the experiments by Khalifa et al. (2013a) for $\mathrm{P} / \mathrm{d}=1.54$, since in 
that case a single tube vibrated due to instability.

As mentioned above, the current simulations predict that the component of the unsteady lift force that is in phase with tube velocity, i.e. the destabilizing force, is mostly generated by the pressure fluctuations on the zones C-C (Fig. 14) of the oscillating tube TV, which correspond to areas in the wake region close to the flow detachment zones. This has some parallelism with the attribution by Granger and Paidoussis (1996) of the force lag to the thin vorticity layer diffused from the vibrating tube surface and then convected along the detached shear layer. According to the current simulations, however, those pressure fluctuations at C-C' are related to the periodic secondary flow induced in the transversal passage between TV and T4, combined with the vortices formed along the central part of the wavy channels below and above TV. For those secondary flows, the areas C-C' behave as intermittent stagnation regions. In turn, those secondary flows are originated by fluid displacement from top and bottom of the oscillating cylinder and by transversal diversion of flow from the main streams due to the changing hydraulic resistance in the wavy channels. In particular, the vortices formed in the main channels below and above tube TV are not really produced by a vortex shedding from the shear layer of the wake of TV in the classical sense, though they are convected downstream.

Certainly, there is a considerable flow re-organization following the tube motion that involves local flow acceleration throughout the domain. This represents significant flow inertia effects, especially in the surroundings of 
the vibrating tube. According to the assumptions of Lever and Weavers theory [1982], the flow inertia effects would manifest as a harmonic perturbation in the cross-section of the main wavy channels with a delay that increases with distance from the oscillating tube, both up and downstream. Qualitatively, this is in line with the measurements by Khalifa et al. (2013b) regarding the phase of velocity fluctuations, as well as with the predictions of the current CFD model, at least downstream. On the other hand, the current computations predict a significant flow exchange between the parallel stream channels at both sides of tube TV, with that transversal flow being determinant in the pattern of pressure fluctuation around TV leading to destabilizing forces. However, no transversal flow has been considered in the Lever and Weaver's models to date. Hence, the development of a new version that could include that transversal flow might contribute to a better description of the flow physics leading to instability and to better predictions.

\section{Conclusions}

The unsteady flow through a parallel triangular array with $\mathrm{P} / \mathrm{d}=1.57$ and one cylinder undergoing forced oscillations in the transverse direction has been studied by means of a 2D URANS CFD model with mesh updating at each time step. After each simulation, the velocity, vorticity and pressure fields computed at successive time steps were FFT processed to obtain the corresponding distribution of fluctuations in amplitude and phase. The predictions obtained lead to the following main conclusions:

- The vibrating tube TV induces vortical secondary flows in the inter- 
cylinder regions with the preceding and the posterior tubes as well as in the adjacent stream channels. Their origin is related to the displacement of fluid from top and bottom of TV and to the transversal diversion of flow from the main streams due to the changing hydraulic resistance in the wavy channels. Those vortices alternate clockwise and anticlockwise rotation during the oscillation cycle, each with a specific phase with respect to tube motion. Besides, vortices in the main channels are convected downstream at an approximately constant speed, whereas the vortices in the wake regions remain fixed.

- Velocity fluctuations are highest along a thin fluid layer adjacent to the vibrating tube. High fluctuation values remain downstream the separation region which is consistent with Khalifa et al.'s measurements [Khalifa et al. (2013a)]. A possible origin is the motion of the separation points on $\mathrm{TV}$ and the high gradient of average velocity across the detached shear layer. At each position, velocity fluctuations are proportional to the tube vibration amplitude amplitude, at least until the highest amplitude tested of $\mathrm{A} / \mathrm{d}=8 \%$, and to the gap velocity if the reduced gap velocity $\mathrm{U}_{R G}>2.5$.

- For $\mathrm{U}_{R G}>1.5$, the periodic secondary flows induced up and downstream from the vibrating tube TV are nearly symmetrical, and so is the pattern of fluctuation amplitude. Besides, the phase of the velocity fluctuations is quite uniform both up and downstream (no vortex convection is revealed downstream), with a jump of $180^{\circ}$ between both sides. This is in agreement with the computations by Hassan and El Bouzidi (2012) and Khalifa et al. (2013b). 
- For $\mathrm{U}_{R G}>1.5$ downstream propagation of flow disturbances was analyzed by using the phase of the vorticity fluctuations, as it determines the speed of vortex convection. Increasing the reduced gap velocity makes the ratio between propagation speed and gap velocity reduce to about 0.4 for $\mathrm{U}_{R G}>5$. This is not far from the 0.52 value reported by [Khalifa et al. (2013a)] from experiments for a relatively similar configuration.

- Khalifa et al.'s measurements [2013a] also showed progressive propagation upstream up to two cylinder rows ahead of TV, whereas the present study predicts a more complex fluctuating pattern with upstream propagation concentrated in the central part of the stream channels at the sides of TV. The discrepancy might indicate an excessive projection of the secondary flow between TV and the preceding cylinder into the main stream channels. It is uncertain to what extent the discrepancy is attributable to the differences between configurations of experiments and computations or to the assumptions of the computational model.

- Around the vibrating tube, highest pressure fluctuations take place on the top and bottom of TV and close to the attachment region, showing good agreement with the instantaneous pressure maps of [Khalifa et al. (2013b)]. Nonetheless, the main responsible for generating a fluctuating lift component in phase with tube velocity, which is a necessary condition for damping-controlled fluidelastic instability, appears to be the pressure fluctuations on the wake side of TV, in particular at the locations where the secondary flow forms periodic stagnation areas. 
- The amplitude of the computed fluctuating lift for a range of reduced velocity shows good agreement with the measurements reported by Sawadogo and Mureithi (2014) for an array with P/d=1.5 under similar conditions. The predicted phase follows the trend of the experimental data too. The calculated fluid-dynamic forces result to be destabilizing for $\mathrm{U}_{R G}>2.3$ (about half the experimental value).

- According to these simulations, the secondary periodic flows that produce the destabilizing forces impose a considerable alternating flow exchange between the two wavy stream channels adjacent to tube TV. The possible inclusion of this transversal flow in a Lever and Weaver's model might contribute to a better description of the systems developing fluidelastic instability.

\section{Acknowledgments}

The authors gratefully acknowledge the financial support received from the Spanish Ministry of Economy and Competitiveness (project MCE-DPI2012-36464) as well as the PhD grant BP-12054 awarded to Ms. de Pedro by the Principality of Asturias Government.

\section{References}

Anderson, B., Hassan, M., Mohany, A., 2014. Modelling of fluidelastic instability in a square inline tube array including the boundary layer effect. Journal of Fluids and Structures. 132 (48), 362-375. 
Chen, S. S., 1983. Instability mechanisms and stability criteria of a group of circular cylinders subjected to cross-flow. part ii: Numerical results and discussion. Journal of Vibrations, Acoustics, Stress, Reliability and Design $105,253-260$.

de Pedro, B., Parrondo, J., Meskell, C., Fernández-Oro, J., 2016. Cfd modelling of the cross-flow through normal triangular tube arrays with one tube undergoing forced vibrations or fluidelastic instability. Journal of Fluids and Structures 64, 6786 .

El Bouzidi, S., Hassan, M., 2015. An investigation of time lag causing fluidelastic instability. Journal of Fluids and Structures 57, 264-276.

Granger, S., Paidoussis, M., 1996. An improvement to the quasi-steady model with application to cross-flow-induced vibration of tube arrays. Journal of Fluid Mechanics 320, 163-184.

Hassan, M., El Bouzidi, S., 2012. Unsteady fluid forces and the time delay in a vibrating tube subjected to cross flow. In Proceedings of the 10th International Conference on Flow-Induced Vibration - FIV 2012 (Eds. G. Bennett and C. Meskell). Dublin, Ireland, 107-114.

Hassan, M., Gerber, A., Omar, H., 2010. Numerical estimation of fluidelastic instability in tube arrays. Journal of Pressure and Vessel Technology 132 (4), 041307 (11 pp.).

Hassan, M., Weaver, D., 2016a. The effects of pitch and mass ratio on transverse and streammwise fluidelastic instability in parallel triangular tube ar- 
rays. In Proceedings of the 11th International Conference on Flow-Induced Vibration and Noise - FIV 2016. The Hague, Nederlands, 107-114.

Hassan, M., Weaver, D., 2016b. Modeling of streamwise and transverse fluidelastic instability in tube arrays. Journal of Pressure Vessel Technology 138.

Khalifa, A., Weaver, D., Ziada, S., 2013a. An experimental study of flowinduced vibration and the associated flow perturbations in a parallel triangular tube array. Journal of Pressure Vessel Technology 135.

Khalifa, A., Weaver, D., Ziada, S., 2013b. Modeling of the phase lag causing fluidelastic instability in a parallel triangular tube array. Journal of Fluids and Structures 43, 371-384.

Lever, J., Weaver, D., 1982. A theoretical model for fluidelastic instability in heat exchanger tube bundles. Journal of Pressure Vessel Technology 104, $147-158$.

Lever, J., Weaver, D., 1986a. On the stability of heat exchanger tube bundles. part i: modified theoretical model. Journal of Sound and Vibration 107, 375-392.

Lever, J., Weaver, D., 1986b. On the stability of heat exchanger tube bundles. part ii: numerical results and comparison with experiment. Journal of Sound and Vibration 107, 393-410.

Li, H., Mureithi, N., 2017. Development of a time delay formulation for fluidelastic instability model. Journal of Fluids and Structures 70, 346359. 
Meskell, C., 2009. A new model for damping controlled fluidelastic instability in heat exchanger tube arrays. Proceedings of the Institution of Mechanical Engineers, Part A: Journal of Power and Energy 223 (4), 361-368.

Paidoussis, M., Mavprilis, D., Price, S., 1984. A potential-flow theory for the dynamics of cylinder arrays in crosss-flow. Journal of Fluid Mechanics 140, $227-252$.

Paidoussis, M. P., Price, S. J., 1988. The mechanisms underlying flow-induced instabilities of cylinder arrays in crossflow. Journal of Fluid Mechanics 187, $45-59$.

Price, S. J., Paidoussis, M. P., 1984. An improved mathematical model for the stability of cylinder rows subject to cross-flow. Journal of Sound and Vibration 97 (4), 615-640.

Sawadogo, T., Mureithi, N., 2014. Fluidelastic instability study in a rotated triangular tube array subject to two-phase cross-flow. part i: Fluid force measurements and time delay extraction. Journal of Fluids and Structures $49(1), 1-15$.

Scott, P., 1987. Flow visualization of cross-flow induced vibrations in tubearrays. Master's Thesis. McMaster University, Hamilton, Ontario, Canada.

Yetisir, M., Weaver, D., 1993a. An unsteady theory for fluidelastic instability in an array of flexible tubes in cross-flow. part i: Theory. Journal of Fluids and Structures 7, 751-766.

Yetisir, M., Weaver, D., 1993b. An unsteady theory for fluidelastic instability 
in an array of flexible tubes in cross-flow. part ii: Results and comparisons with experiments. Journal of Fluids and Structures 7, 767-782. 
a)

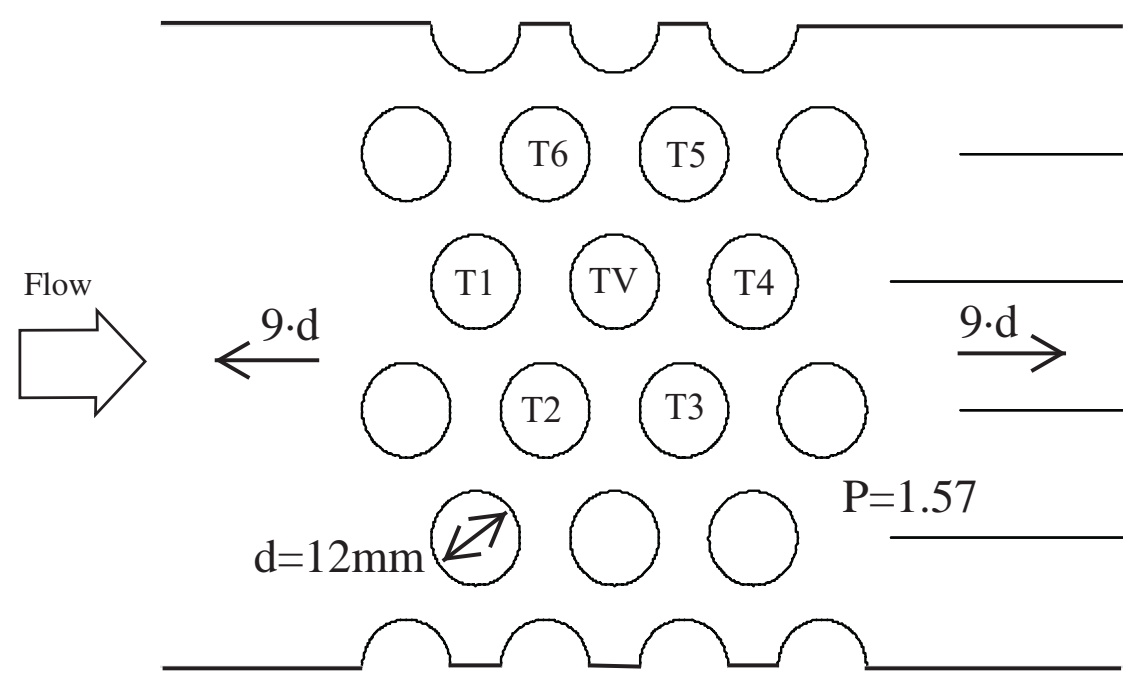

b)

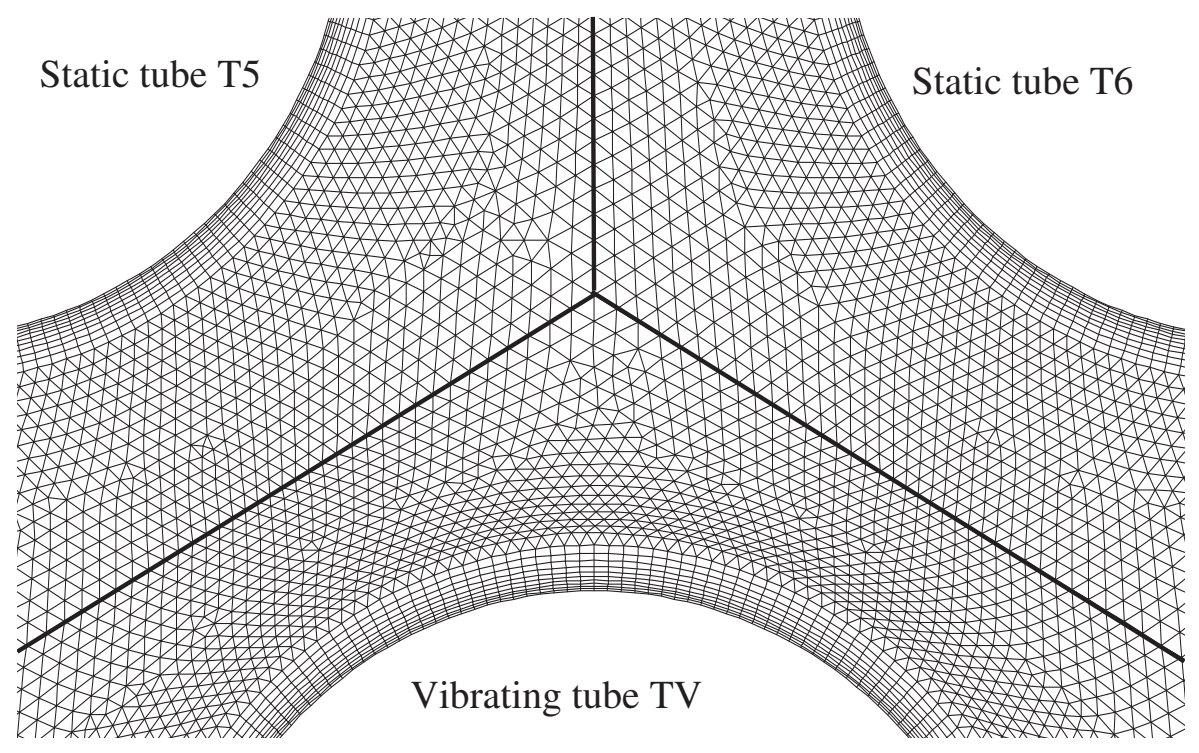

Figure 1: Computational domain. a) Array lay-out. b) Detail of mesh between tubes with tube TV shifted $8 \%$ d upwards. 


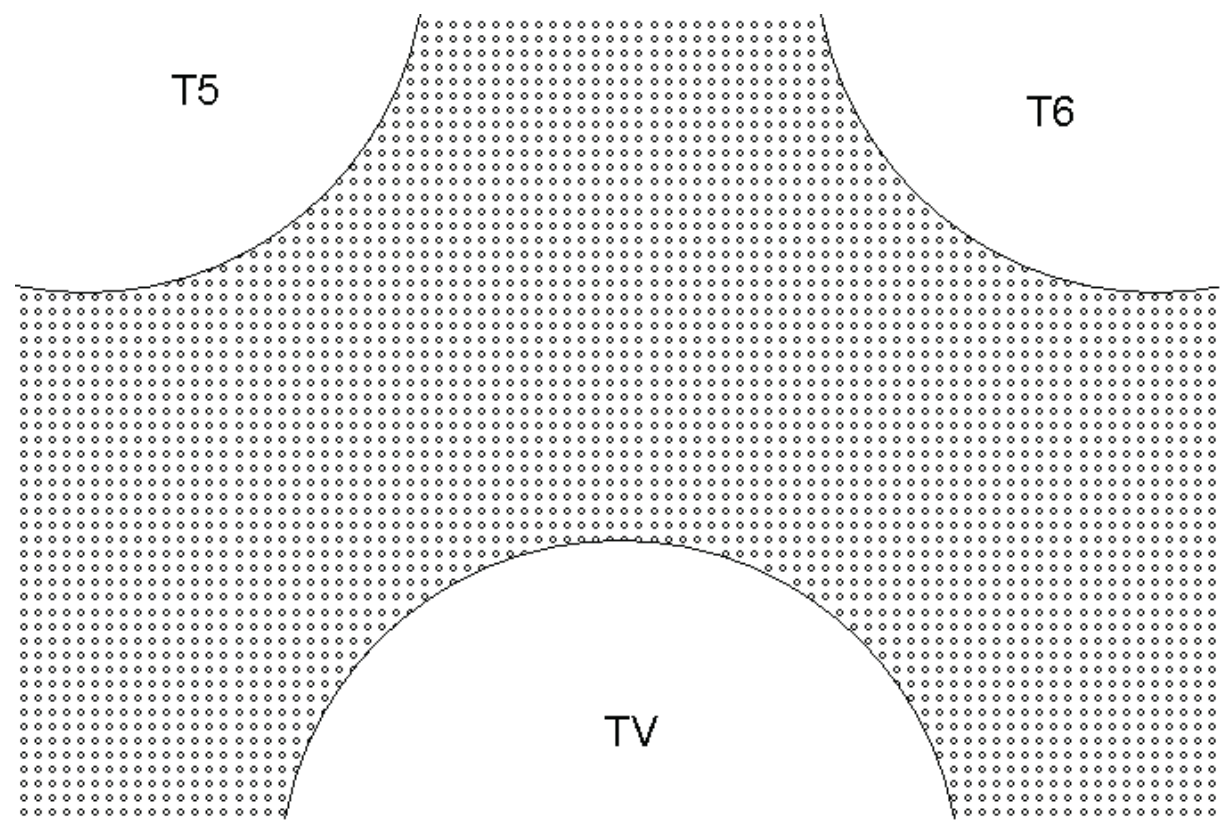

Figure 2: Detail of the regular grid used for FFT post-processing of CFD data. 
a)

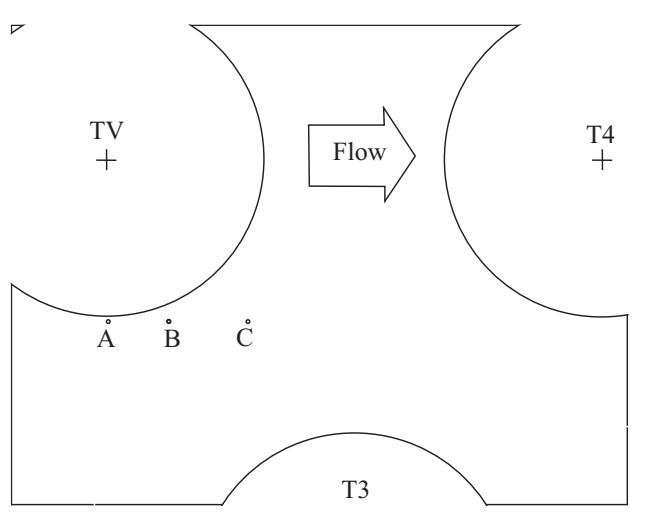

b)
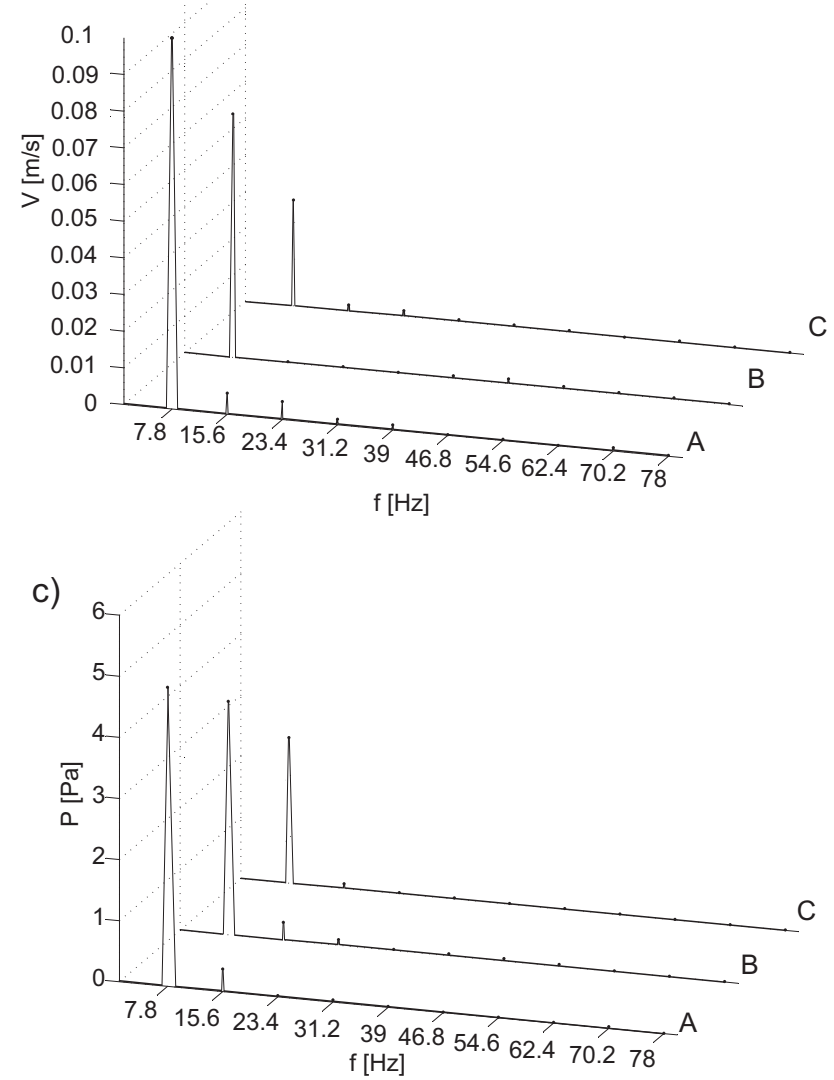

Figure 3: Monitored positions (a) and amplitude spectra of velocity (b) and pressure (c). Main system parameters: $\mathrm{U}_{U}=0.21 \mathrm{~m} / \mathrm{s}, \mathrm{A} / \mathrm{d}=0.83 \%, \mathrm{f}=7.81 \mathrm{~Hz}\left(\mathrm{U}_{R G}=5.34\right)$. 
a)

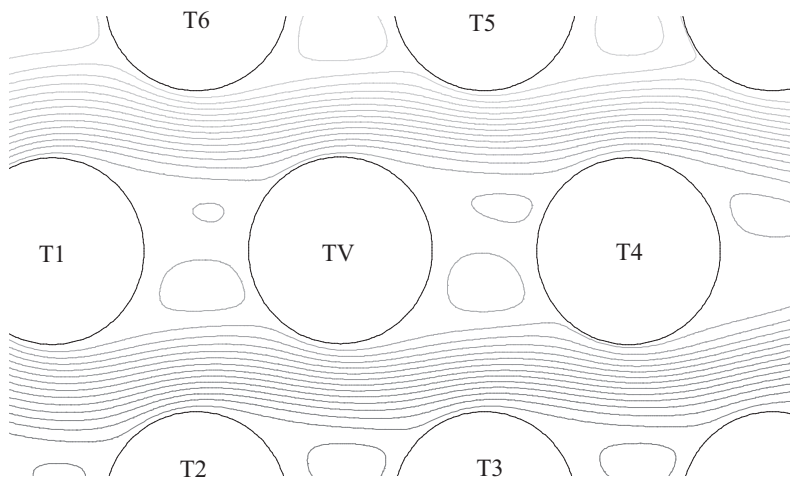

b)

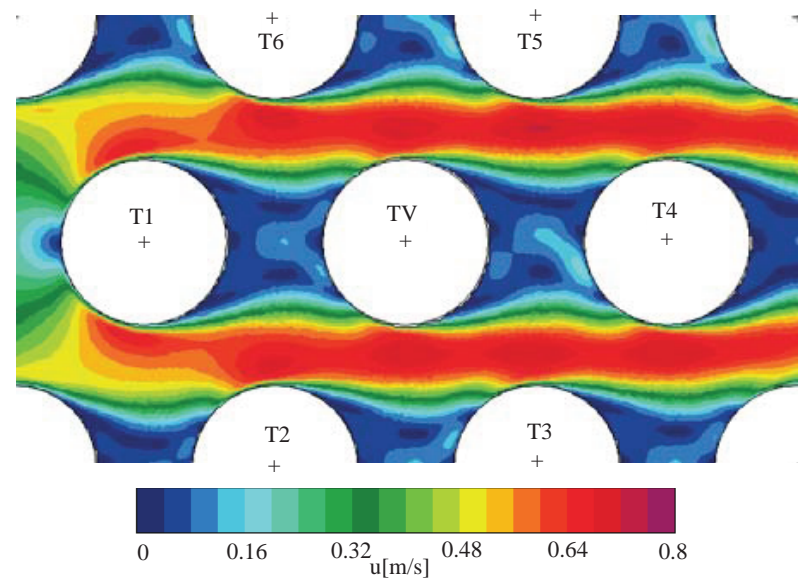

c)

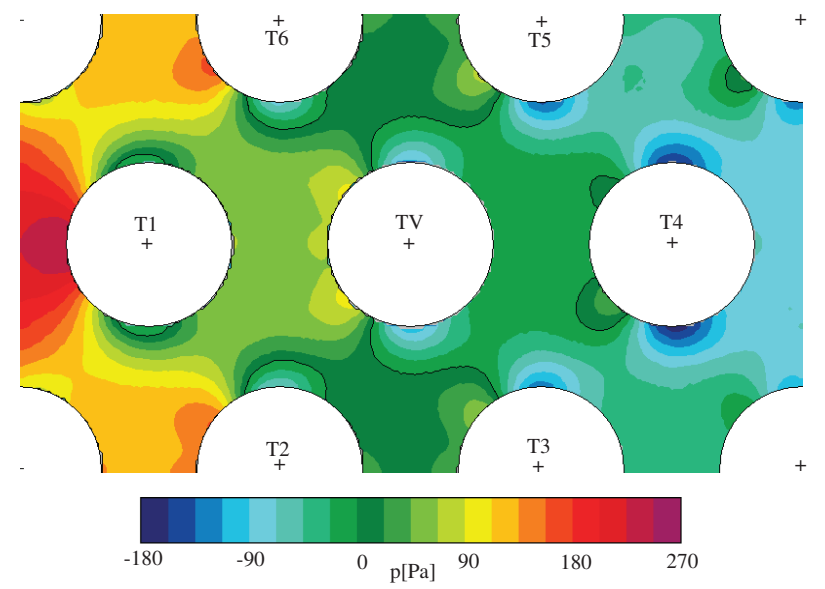

Figure 4: Average flow: streamlines (a), velocity (b) and pressure (c). System parameters as in Fig. 3. 


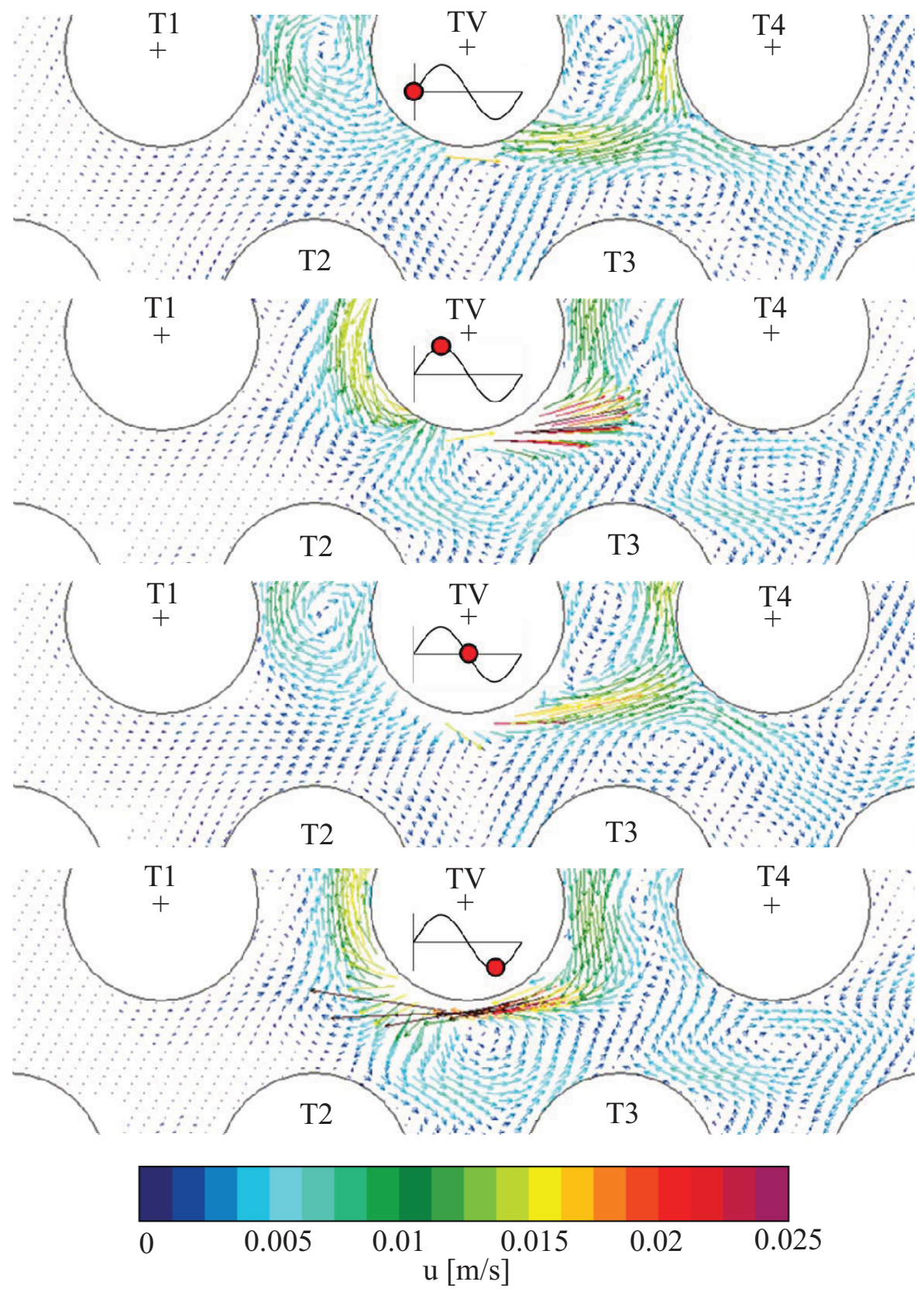

Figure 5: Instantaneous fluctuation of the velocity vector with tube TV at four positions $\left(0^{\circ}, 90^{\circ}, 180^{\circ}\right.$ and $\left.270^{\circ}\right)$ during one oscillation. System parameters as in Fig. 3 
a)

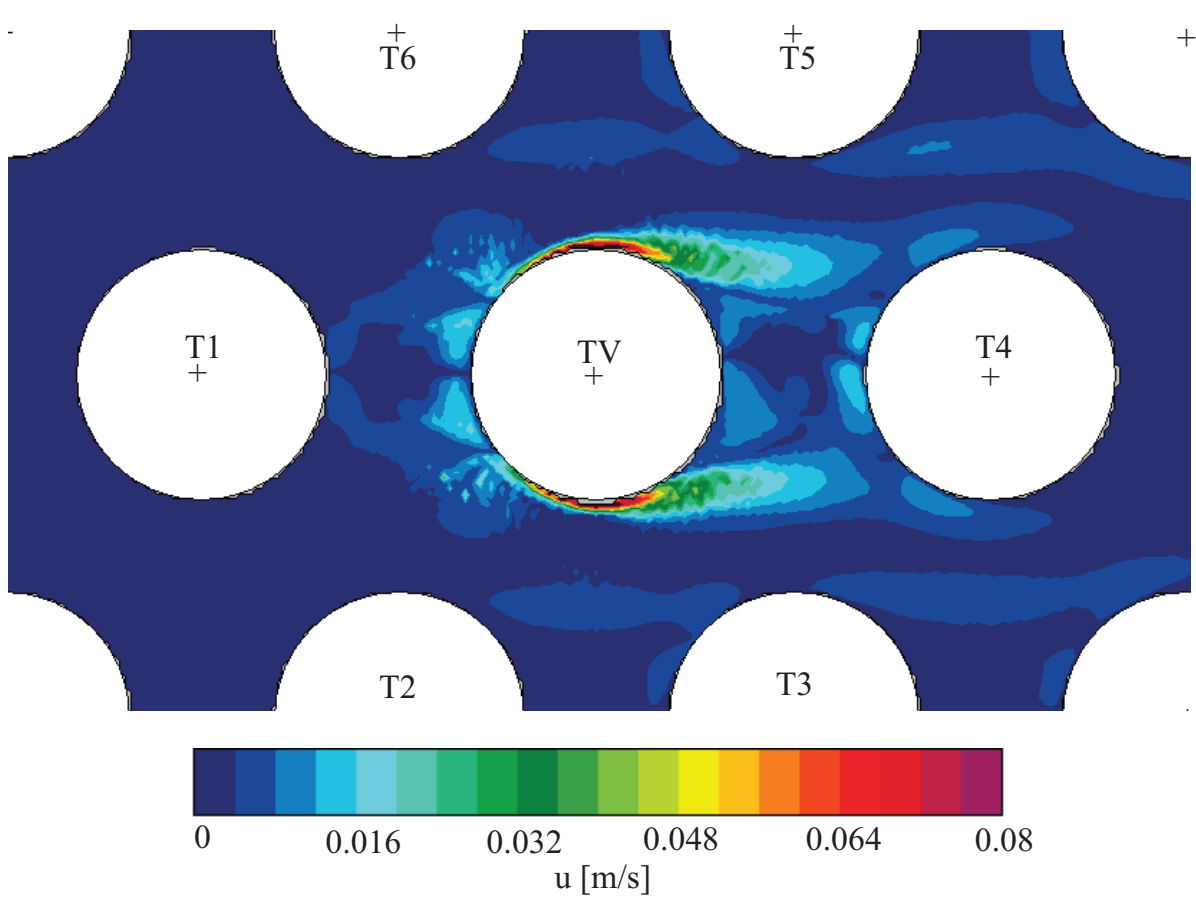

b)

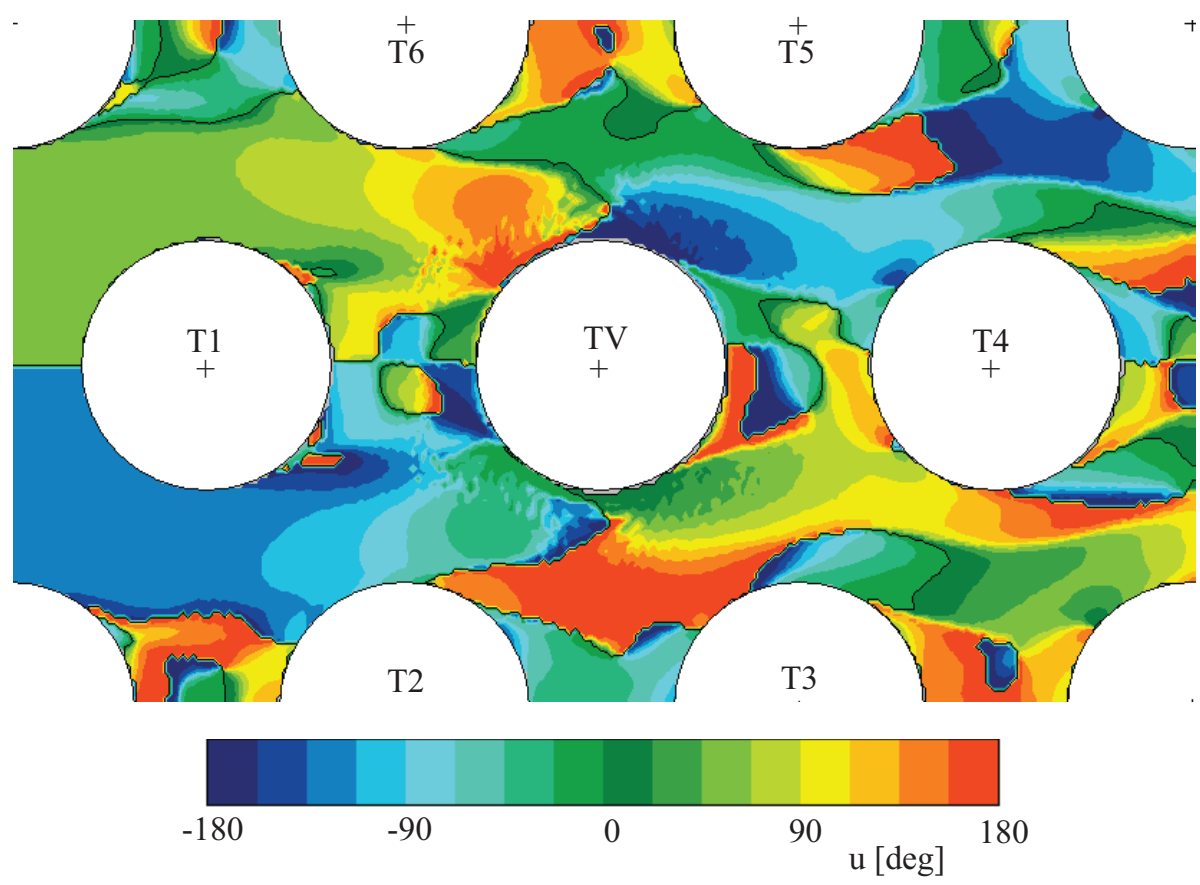

Figure 6: Amplitude (a) and phase (b) of the velocity fluctuations at the $1^{\text {st }}$ harmonic. System parameters as in Fig. 3 

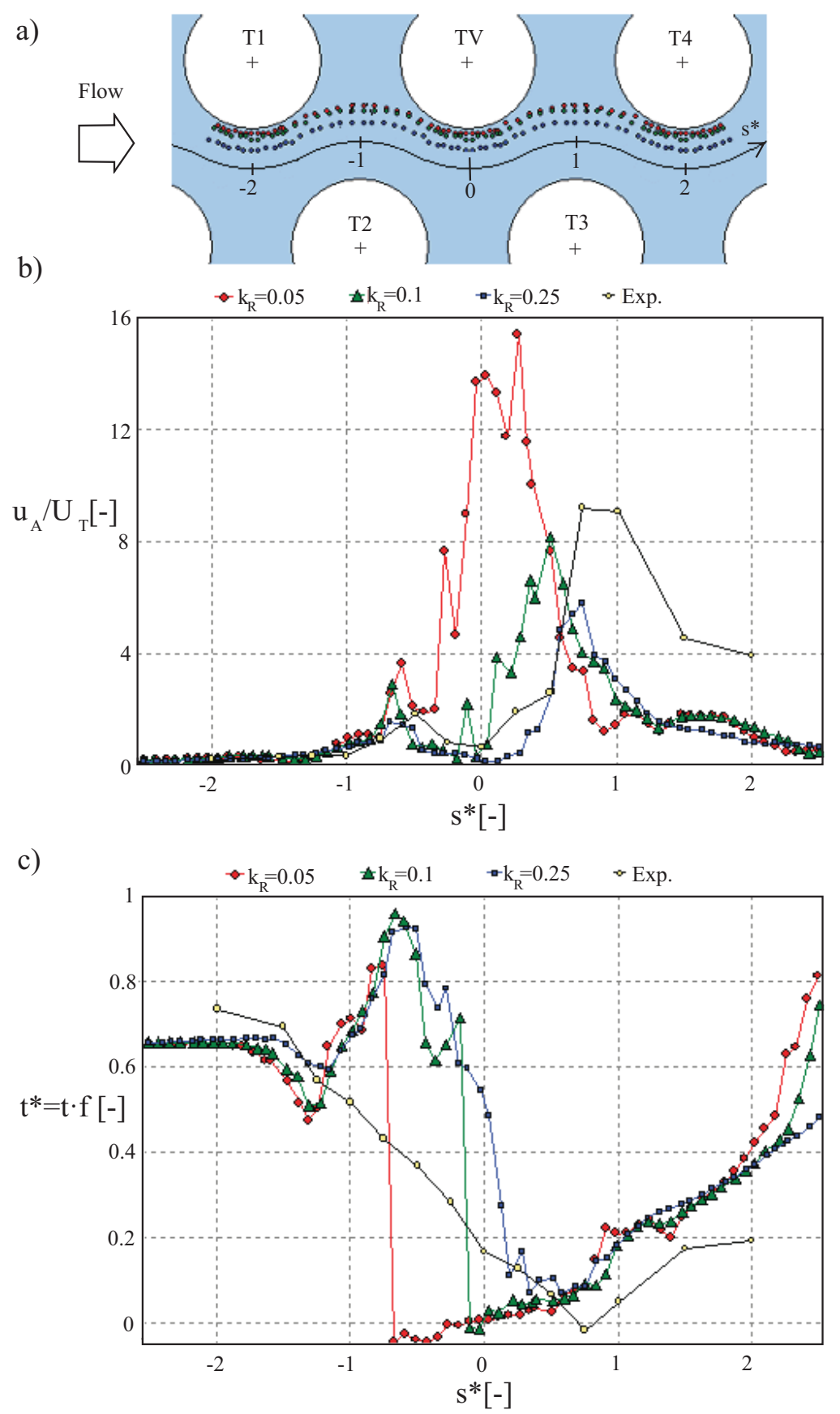

Figure 7: Velocity fluctuations along three fluid lines (system parameters as in Fig. 3). Experimental data from [6]. a) Fluid lines. b) Velocity amplitude normalized by tube velocity. c) Time delay normalized by oscillation period. 
a)

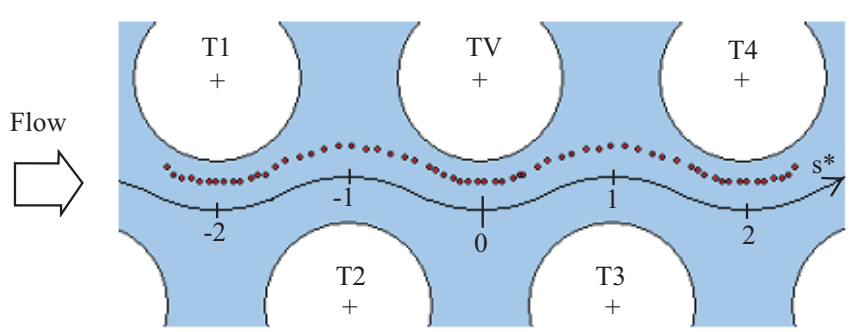

b)
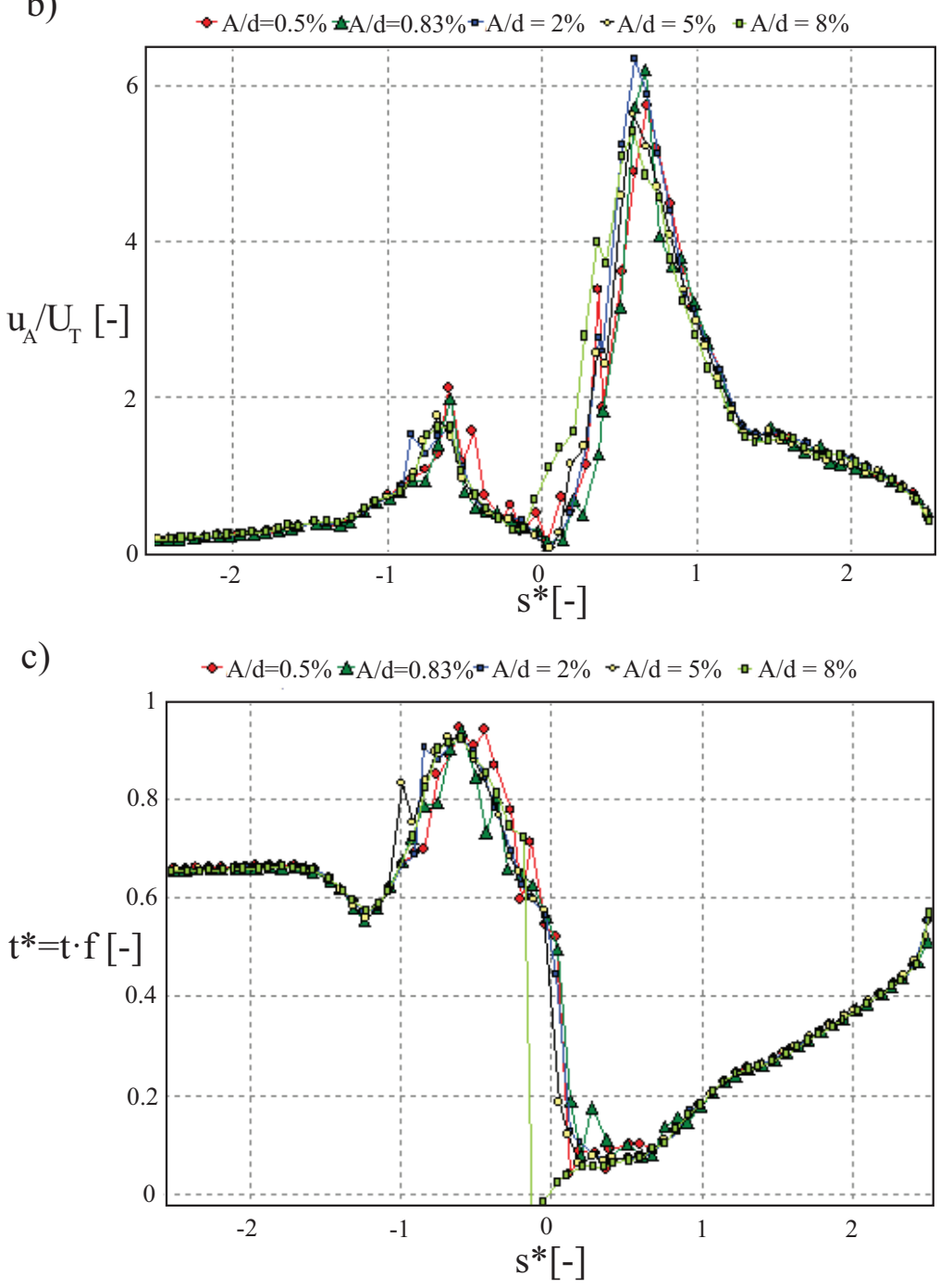

Figure 8: Velocity fluctuations along one fluid line for several oscillation amplitudes $\left(\mathrm{U}_{R G}=5.34 \mathrm{~m} / \mathrm{s}, \mathrm{f}=7.81 \mathrm{~Hz}\right)$. a) Fluid line $\left(\mathrm{k}_{R}=0.2\right)$. b) Velocity amplitude normalized by tube velocity. c) Time delay normalized by oscillation period. 
a)

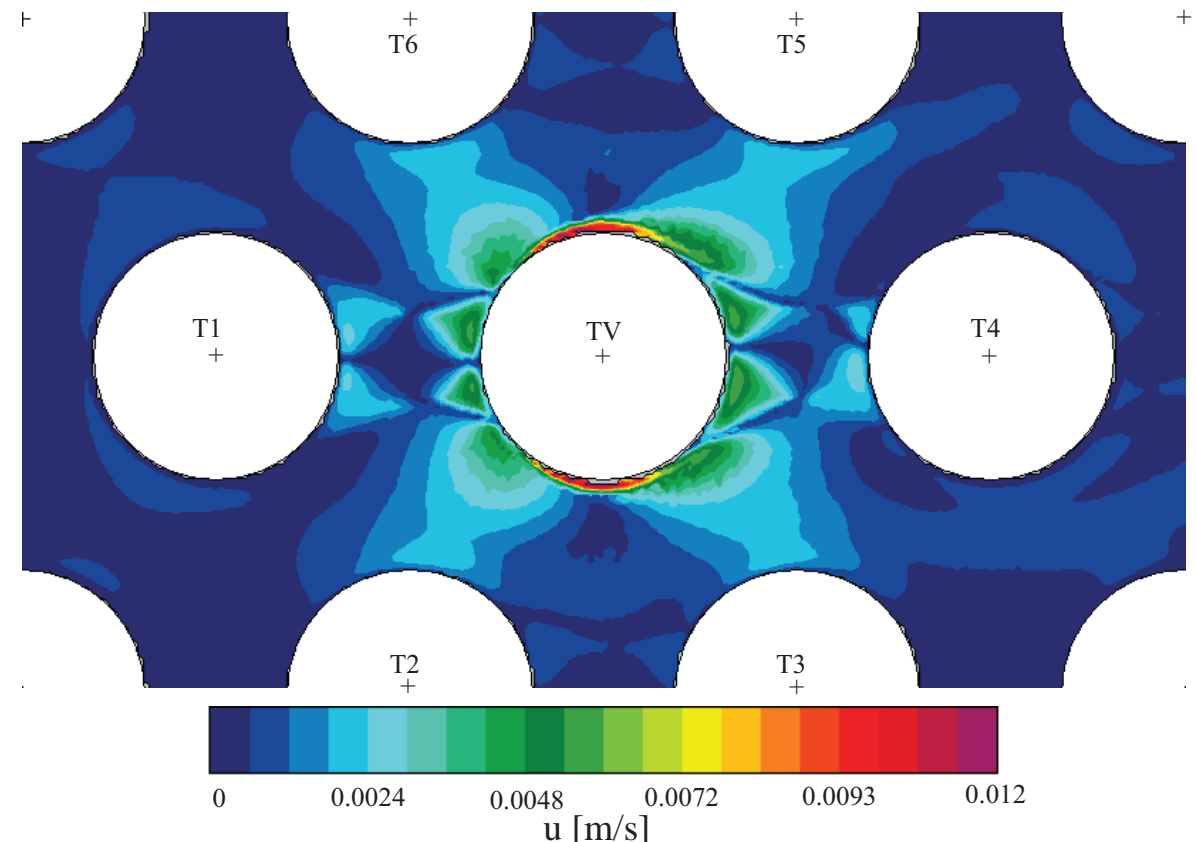

b)

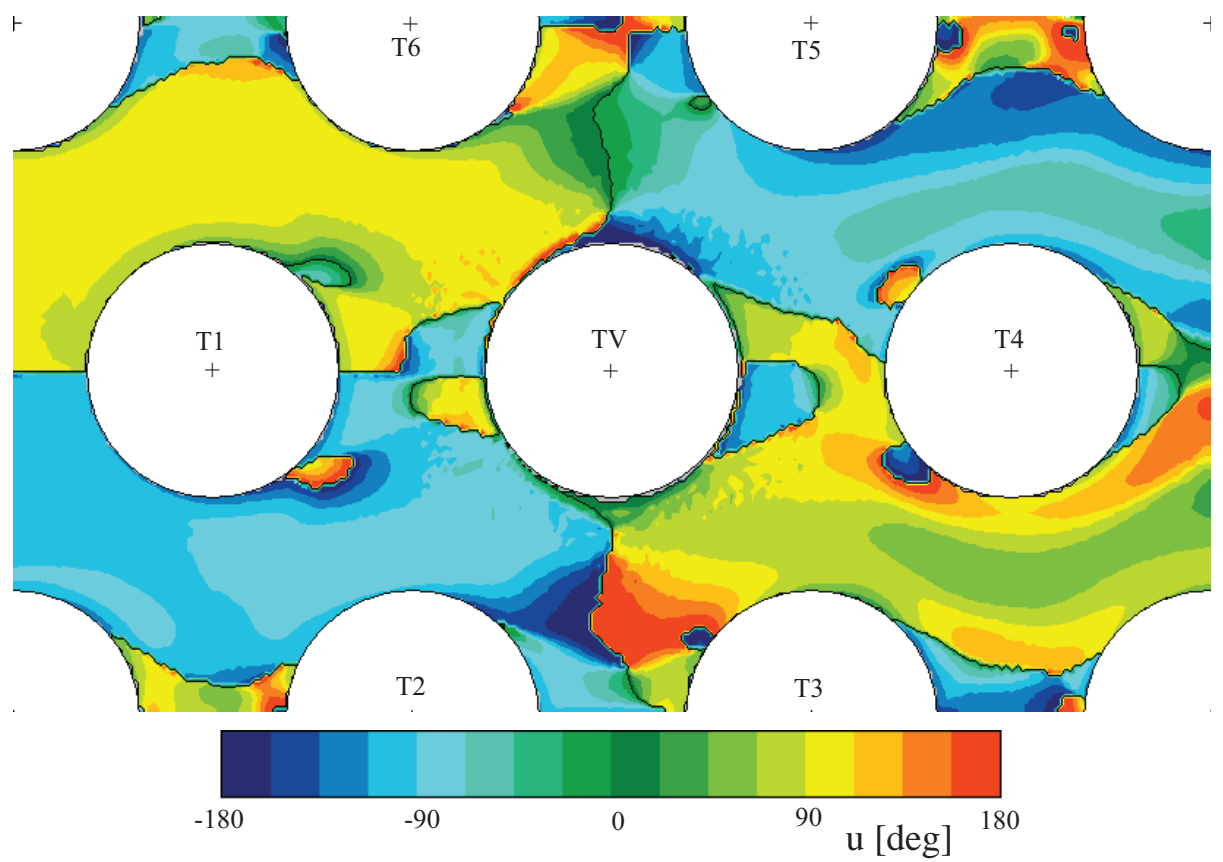

Figure 9: Amplitude (a) and phase (b) of the velocity fluctuations at the $1^{\text {st }}$ harmonic $\left(\mathrm{U}_{U}=0.03 \mathrm{~m} / \mathrm{s}\right.$, other system parameters as in Fig. 3). 
a)

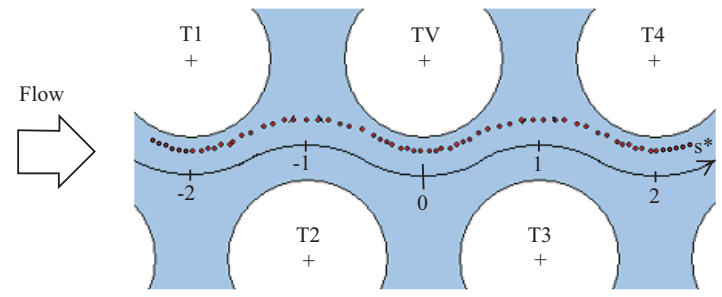

b) $\quad 0.06 \quad * \mathrm{U}_{\mathrm{R}} \overline{\overline{\mathrm{G}}} 0.76 \quad \nexists \mathrm{U}_{\mathrm{R}} \overline{\overline{\mathrm{G}}} 2.29 \quad * \mathrm{U}_{\mathrm{R}} \overline{\overline{\mathrm{G}}} 3.56 * \mathrm{U}_{\mathrm{R}}=5.34 * \mathrm{U}_{\mathrm{R} \overline{\mathrm{G}}}=8.01$
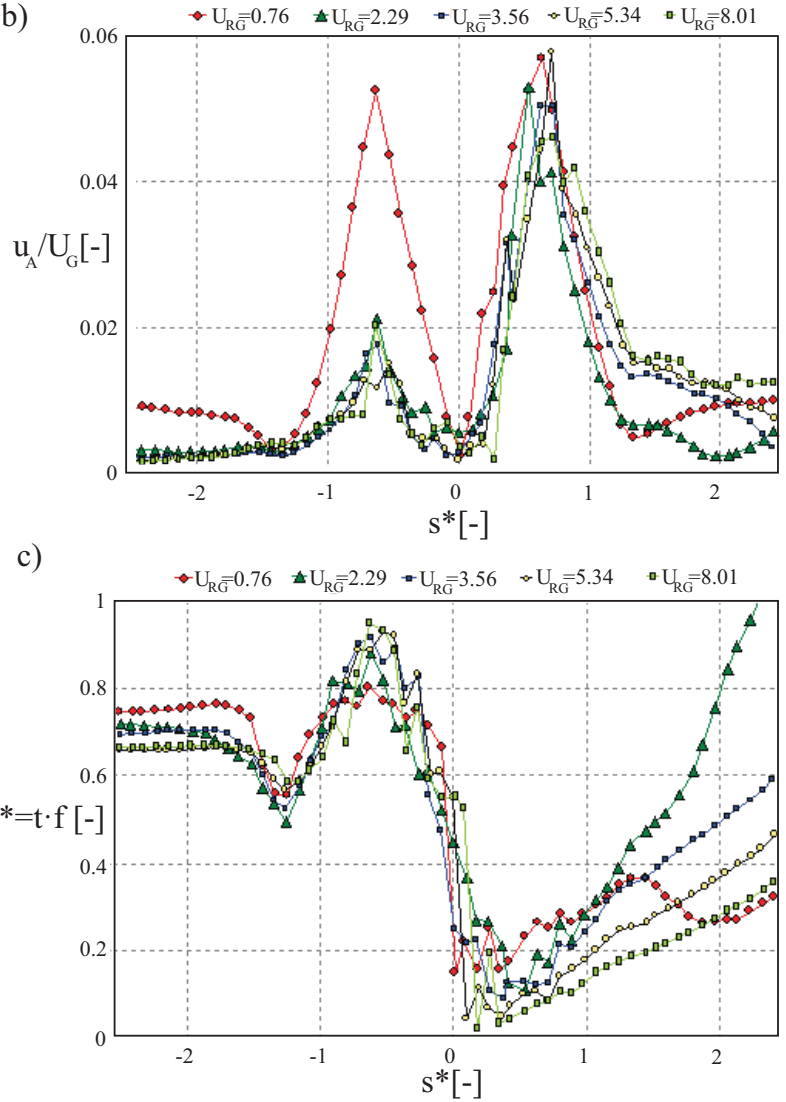

Figure 10: Velocity fluctuations along one fluid line for several reduced gap velocities $\mathrm{U}_{R G}(\mathrm{~A}=0.83 \% \mathrm{~d}, \mathrm{f}=7.81 \mathrm{~Hz})$. a) Fluid line $\left(\mathrm{k}_{R}=0.2\right)$. b) Velocity amplitude normalized by gap velocity. c) Normalized time delay. 


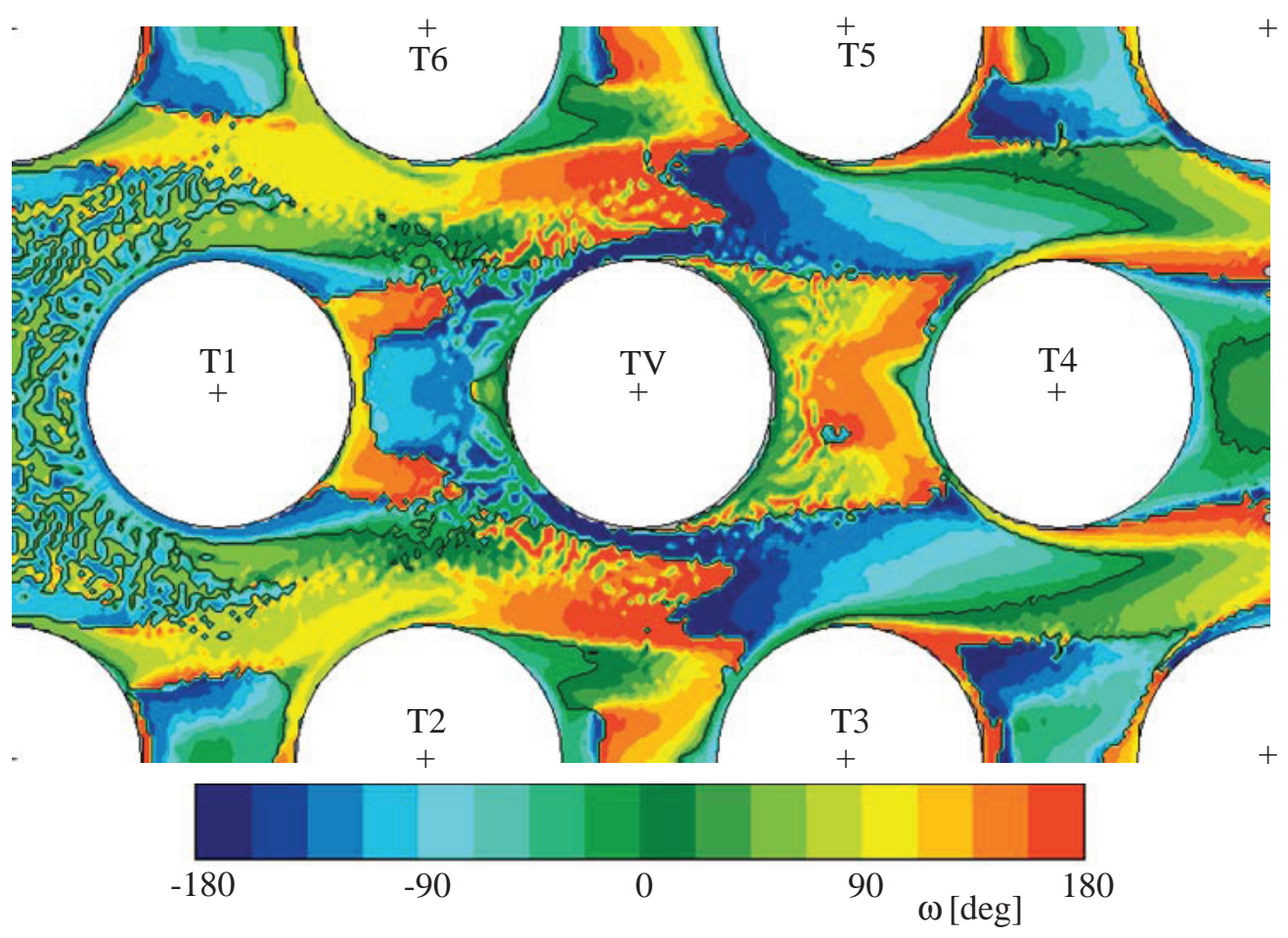

Figure 11: Phase of the vorticity fluctuations at the $1^{\text {st }}$ harmonic. System parameters as in Fig. 3. 
a)

b)
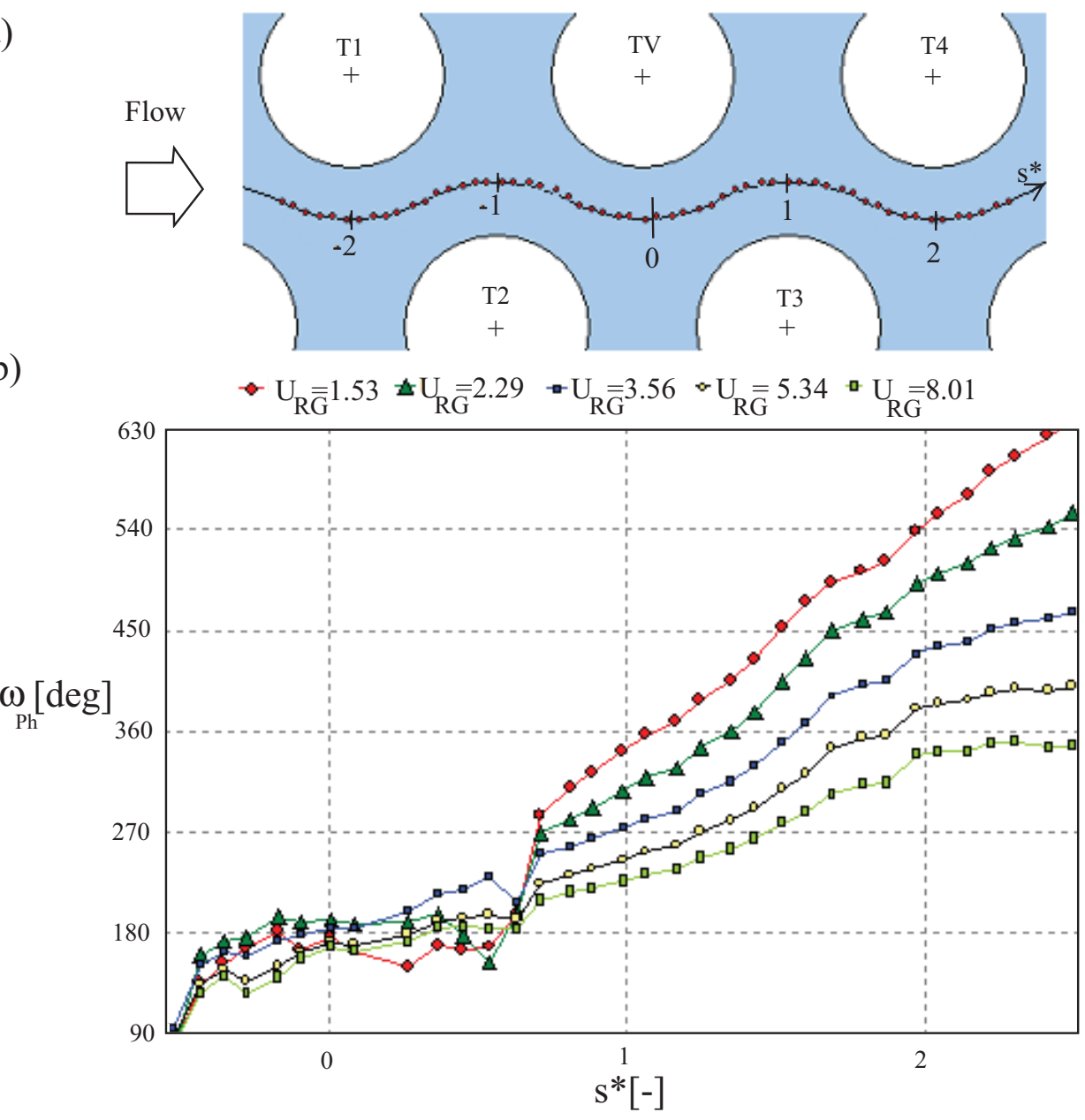

Figure 12: Phase of vorticity fluctuations along the central fluid line for several reduced gap velocities. $\mathrm{U}_{R G}(\mathrm{~A}=0.83 \% \mathrm{~d}, \mathrm{f}=7.81 \mathrm{~Hz})$. a) Fluid line $\left(\mathrm{k}_{R}=0.5\right)$. b) Phase delay. 


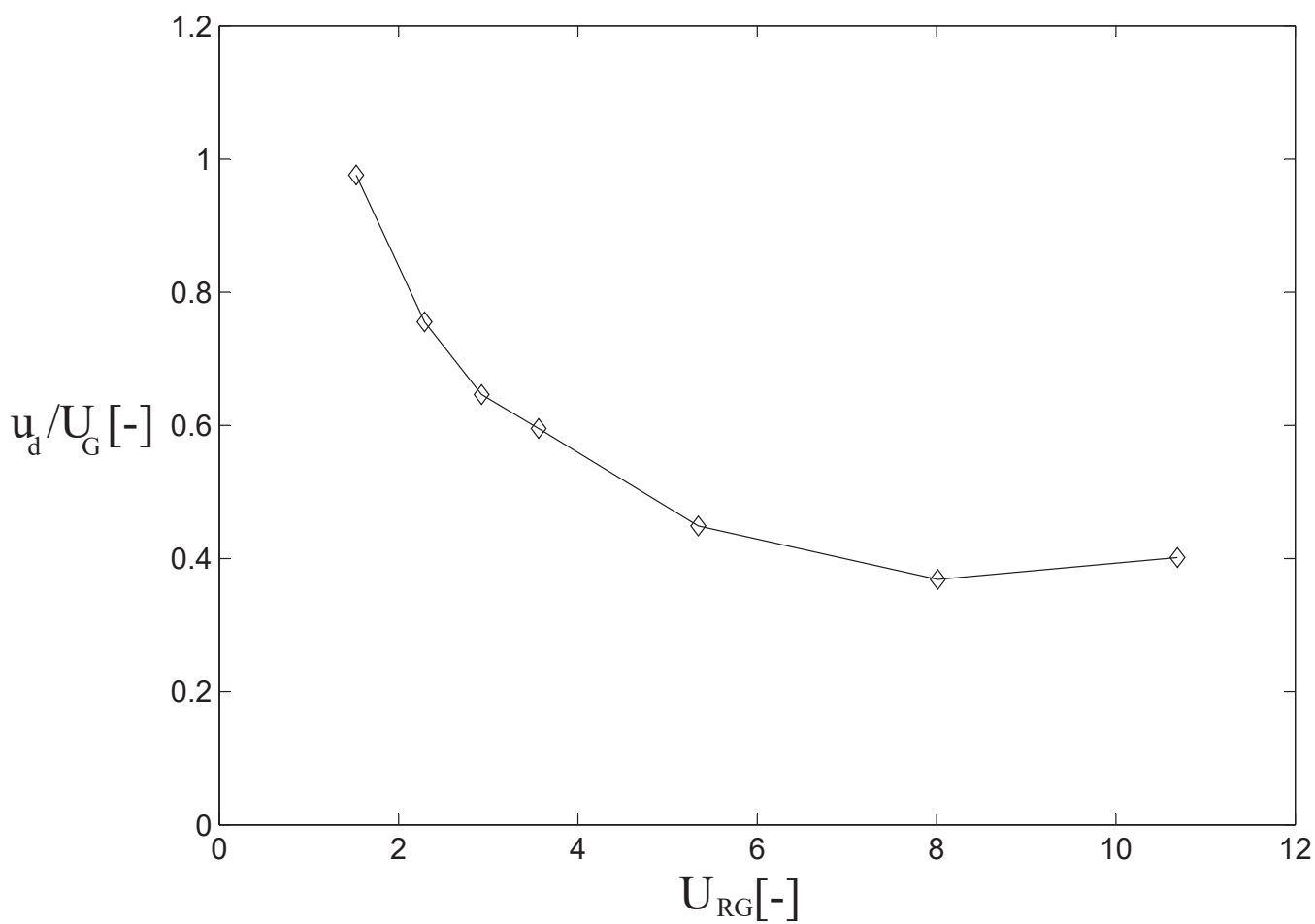

Figure 13: Speed of propagation of vorticity fluctuations downstream from tube TV, normalized by gap velocity. 
a)
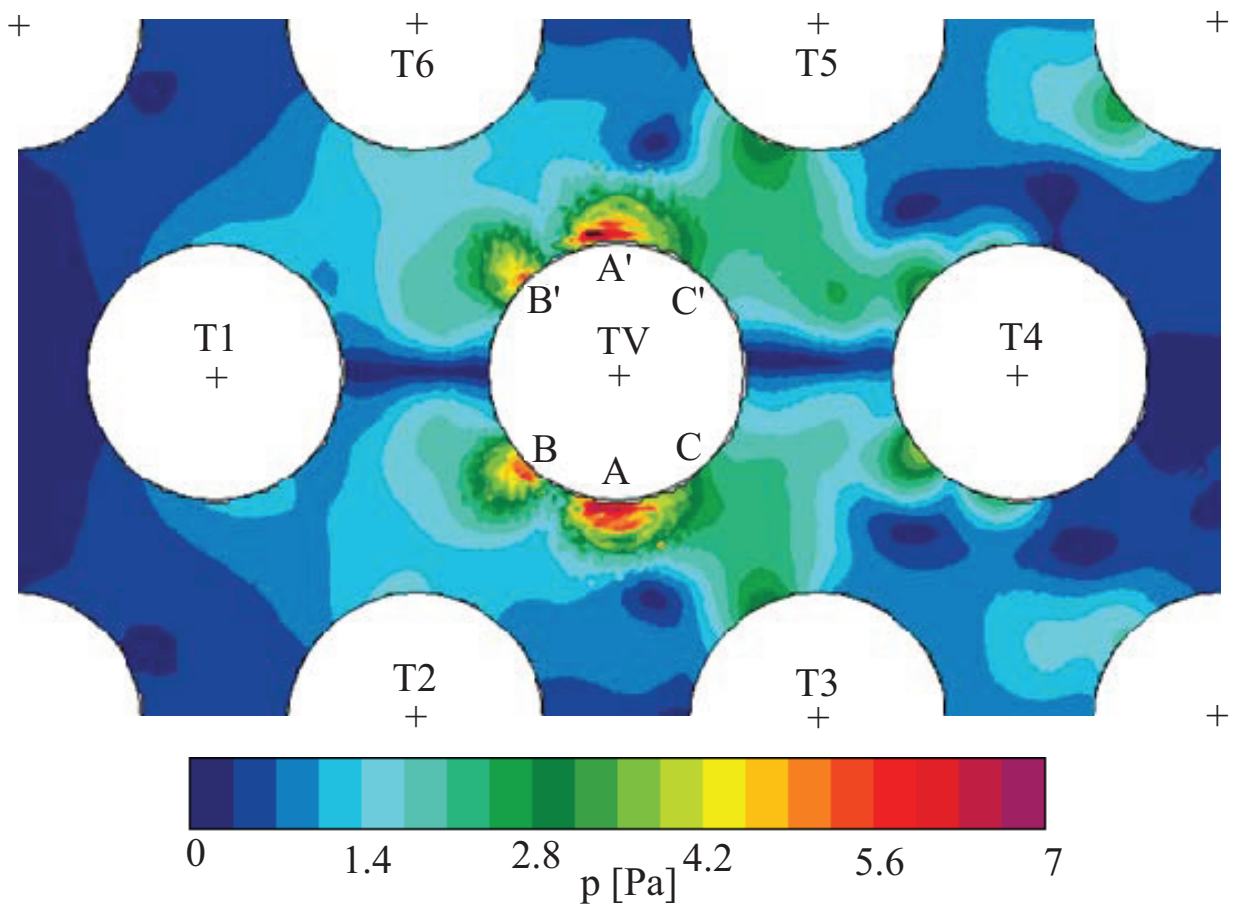

b)
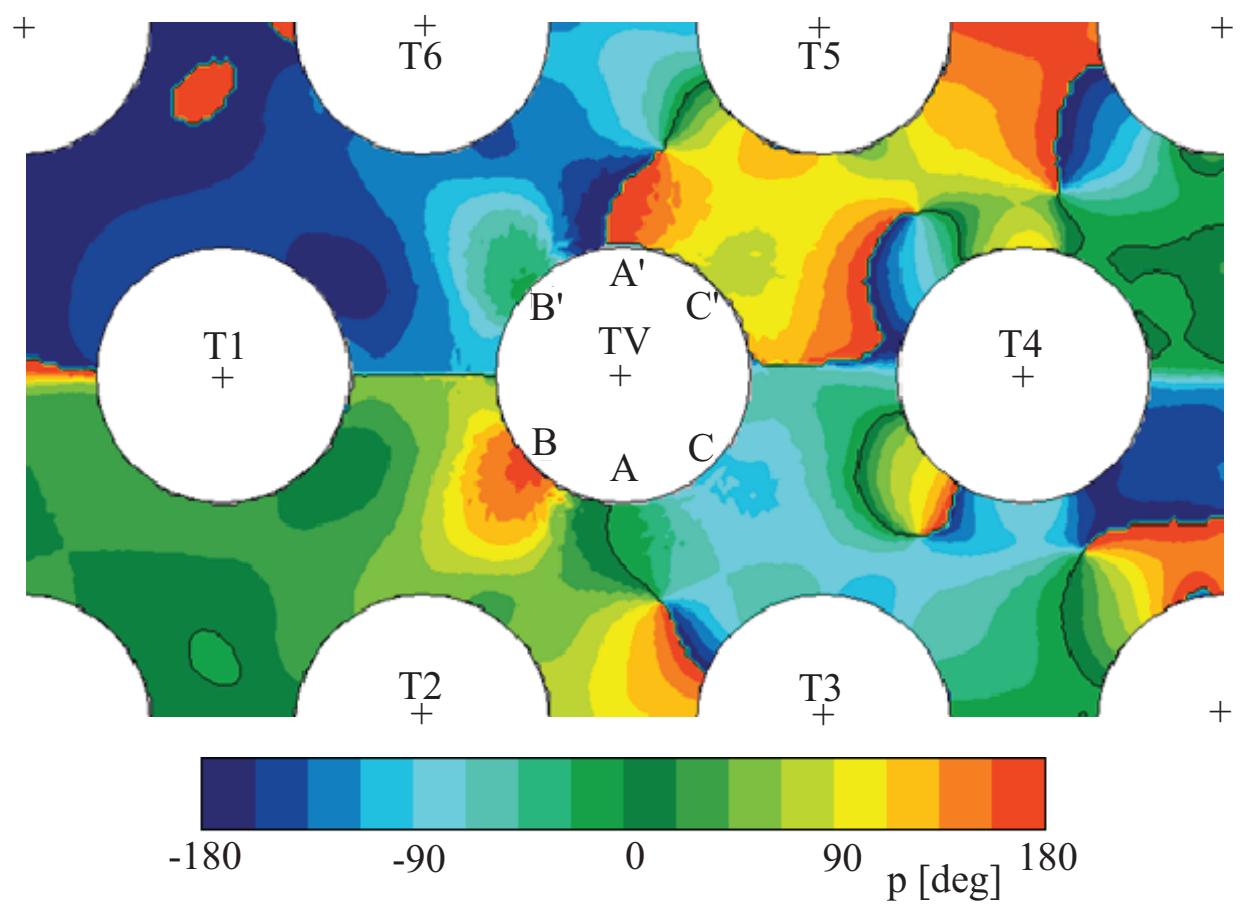

Figure 14: Amplitude (a) and phase (b) of the pressure fluctuations at the $1^{\text {st }}$ harmonic. System parameters as in Fig. 3 
a)

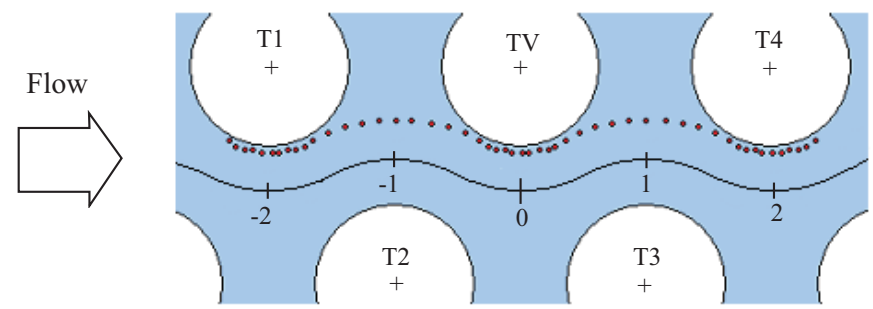

b)

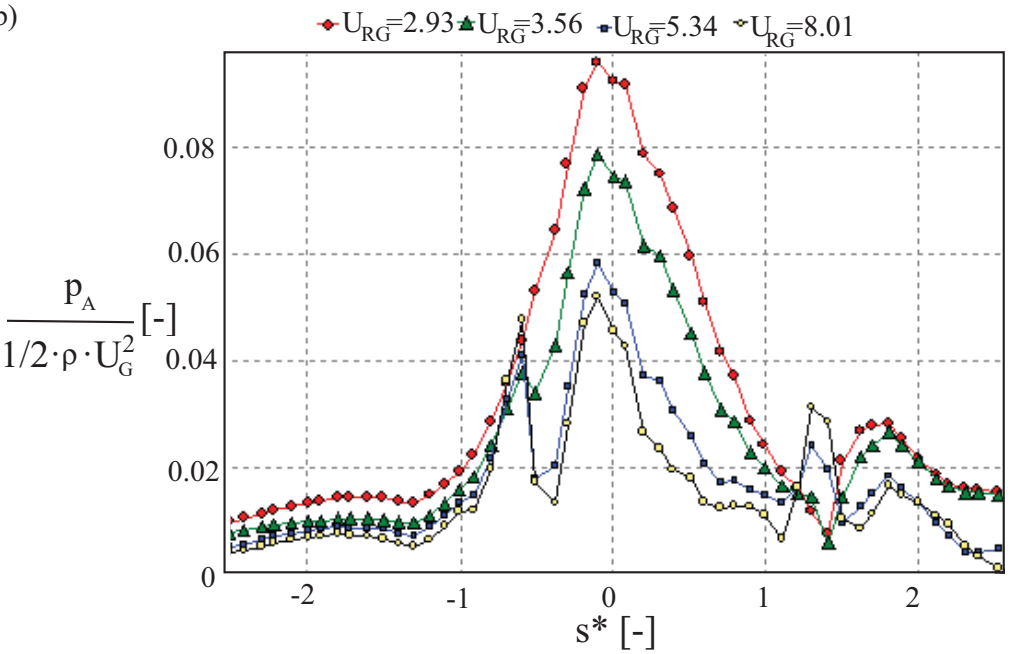

c)

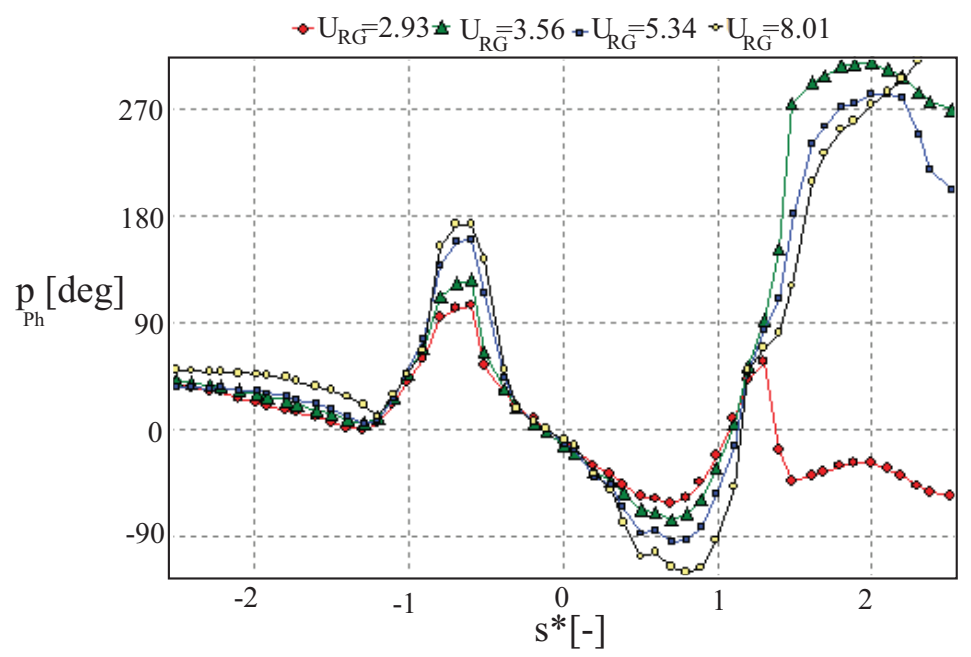

Figure 15: Pressure fluctuations along one fluid line for several reduced gap velocities $\mathrm{U}_{R G}(\mathrm{~A}=0.83 \% \mathrm{~d}, \mathrm{f}=7.81 \mathrm{~Hz})$. a) Fluid line $\left(\mathrm{k}_{R}=0.05\right)$. b) Pressure amplitude normalized by gap dynamic pressure. c) Phase of pressure fluctuations. 

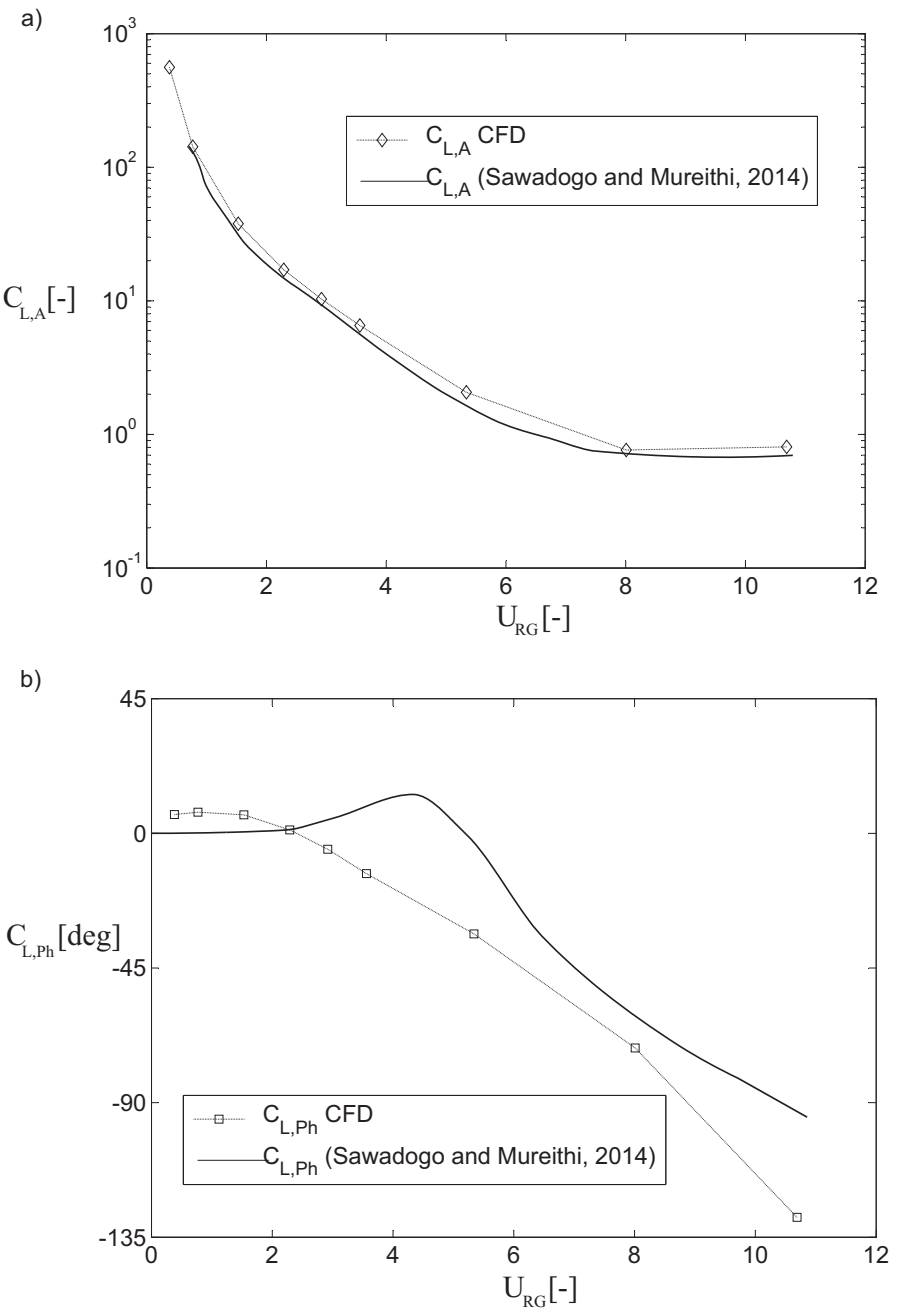

Figure 16: Modulus and phase (relative to position of tube TV) of the lift coefficient as a function of the reduced gap velocity:

$$
C_{L}=\frac{F_{L}}{\frac{1}{2} \rho U_{G}^{2} d}
$$

Recently, Li and Mureithi (2017) explored the determination of that time delay function based on a frequency approach in the form of an equivalent Theodorsen function. 